\title{
Stochastic Target Problems with Controlled Loss*
}

\author{
Bruno Bouchard \\ CEREMADE, Université Paris Dauphine \\ and CREST-ENSAE \\ bouchard@ceremade.dauphine.fr
}

\author{
Romuald Elie \\ CEREMADE, Université Paris Dauphine \\ and CREST-ENSAE \\ elie@ceremade.dauphine.fr
}

\author{
Nizar Touzi \\ Ecole Polytechnique Paris \\ Centre de Mathématiques Appliquées \\ touzi@cmap.polytechnique.fr
}

This version: September 2008

\begin{abstract}
We consider the problem of finding the minimal initial data of a controlled process which guarantees to reach a controlled target with a given probability of success or, more generally, with a given level of expected loss. By suitably increasing the state space and the controls, we show that this problem can be converted into a stochastic target problem, i.e. find the minimal initial data of a controlled process which guarantees to reach a controlled target with probability one. Unlike the existing literature on stochastic target problems, our increased controls are valued in an unbounded set. In this paper, we provide a new derivation of the dynamic programming equation for general stochastic target problems with unbounded controls, together with the appropriate boundary conditions. These results are applied to the problem of quantile hedging in financial mathematics, and are shown to recover the explicit solution of Föllmer and Leukert [5].
\end{abstract}

Key words: Stochastic target problem, discontinuous viscosity solutions, quantile hedging.

AMS 1991 subject classifications: Primary 49L25, 60J60; secondary 49L20, 35K55.

\footnotetext{
${ }^{*}$ This research is part of the Chair Financial Risks of the Risk Foundation sponsored by Société Générale, the Chair Derivatives of the Future sponsored by the Fédération Bancaire Française, the Chair Finance and Sustainable Development sponsored by EDF and Calyon, and the Chair Les particuliers face au risque sponsored by Groupama.
} 


\section{Introduction}

For $0 \leq t \leq T$, we are given two controlled diffusion processes $\left\{X_{t, x}^{\nu}(s), t \leq s \leq T\right\}$ and $\left\{Y_{t, x, y}^{\nu}(s), t \leq s \leq T\right\}$ with values respectively in $\mathbb{R}^{d}$ and $\mathbb{R}_{+}$, satisfying the initial condition $\left(X_{t, x}^{\nu}, Y_{t, x, y}^{\nu}\right)(t)=(x, y)$. The main objective of this paper is to study the stochastic target problem with controlled probability of success:

$$
\bar{V}(t, x, p):=\inf \left\{y \geq 0: \mathbb{P}\left[Y_{t, x, y}^{\nu}(T) \geq g\left(X_{t, x}^{\nu}(T)\right)\right] \geq p \text { for some admissible control } \nu\right\} \text {. }
$$

In the case $p=1, V(t, x):=\bar{V}(t, x, 1)$ reduces to the stochastic target problem studied in Soner and Touzi $[9,10]$, who concentrate on the case where the control $\nu$ takes values in a bounded set.

For $p<1$, this problem was introduced in the context of financial mathematics by Föllmer and Leukert [5]. In the latter paper, the process $X$ models the price of some given securities and is not affected by the control $\nu$ which corresponds to the portfolio strategy of the investor. The process $Y$ represents the value of the investor's portfolio and is defined by a diffusion whose coefficients are linear in the control variable. In this special context, Föllmer and Leukert [5] use a duality argument to convert this problem into a classical test problem in mathematical statistics. An elegant solution is then obtained by a direct application of the Neyman-Pearson Lemma. This approach applies in the case where the securities prices are driven by general semimartingales but the linearity in the control is crucial in order to use their duality argument.

In particular, the duality approach of [5] does not extend to the general nonlinear controlled diffusion case.

Note that a possible approach consists in introducing the standard stochastic control problem:

$$
p(t, x, y):=\max _{\nu} \mathbb{P}\left[Y_{t, x, y}^{\nu}(T) \geq g\left(X_{t, x}^{\nu}(T)\right)\right]
$$

which corresponds to the inverse of $V(t, x, p)$ with respect to the $p$-variable, i.e. $p(t, x, \bar{V}(t, x, p))=$ $p$. Then, one can provide a characterization of the value function $p(\cdot)$ by the dynamic programming approach (DPE), and obtain $\bar{V}$ by inverting the solution with respect to the $y$-variable. We may also translate the DPE for $p(t, x, y)$ into a partial differential equation (PDE) for $\bar{V}$ by using the above relation. This however requires some regularity of the value function $p(t, x, y)$ which is apriori difficult to prove. One could also solve numerically the PDE associated to $p(\cdot)$ and then invert it numerically. This would nonetheless require the computation of $p(\cdot)$ on a large grid of different values $y$ and introduce an additional error due to the numerical inversion.

In this paper, we propose a direct treatment of the problem $\bar{V}$ along the lines of [9]. The keyidea is to convert the problem $\bar{V}$ into a stochastic target problem by diffusing the probability of reaching the target and considering it as an additional controlled state variable $P^{\alpha}$. This is a direct consequence of the martingale representation theorem in our assumed Brownian filtration. This

reformulation of the problem $\bar{V}$ opens the door to the geometric dynamic programming approach of [9], but raises additional difficulties. First, the additional control process in the increased state is unbounded leading to a singular stochastic target problem. Second, the re-formulation is crucially subjet to the state constraint $P^{\alpha} \in[0,1]$ a.s. which leads to non-trivial boundary conditions. 
Our first main result is an extension of the derivation of the dynamic programming equation of [9] to the general case where the control takes values in an unbounded set. This is achieved by conveniently introducing a semilimit relaxation of the corresponding natural dynamic programming equation. While the subsolution derivation follows the lines of the original argument of [9], we provide a new method for the derivation of the supersolution property which does not require any compactness, and avoids delicate passages to the limits. We also provide a description of the terminal condition in the present unbounded control case.

Our second main result concerns the special case of the stochastic target problem with controlled probability of success $\bar{V}$. Under fairly general conditions, we show that the state constraint on $P^{\alpha}$ yields the natural boundary conditions $\bar{V}(t, x, 1-)=V(t, x)$ and $\bar{V}(t, x, 0+)=0$ for $t<T$. At the final time $T$, there is however no clear guess of what should be the behaviour of $\bar{V}$. Under some extra conditions, we prove that $\bar{V}(T-, x, p)=p g(x)$ which is a "face-lifted" version of the natural boundary condition $g(x) \mathbf{1}_{p>0}$.

Notice that the geometric dynamic programming approach of this paper extends to a larger class of problem, namely to stochastic target problems with controlled loss:

$$
V^{\ell}(t, x, p):=\inf \left\{y \geq 0: \mathbb{E}\left[\ell \circ G\left(X_{t, x}^{\nu}(T), Y_{t, x, y}^{\nu}(T)\right)\right] \geq p \text { for some admissible } \nu\right\},
$$

where $G(x, y)$ is non-decreasing in $y$ and the loss function $\ell$ is non-decreasing. The above problem $\bar{V}$ corresponds to the special case $\ell:=\mathbf{1}_{\mathbb{R}_{+}}$.

Finally, we apply our result to the so-called quantile hedging example of Föllmer and Leukert [5]. By using the supersolution property of $\bar{V}$, we reproduce the explicit solution of [5] in the complete market case. The key-idea is to observe that the convex conjugate function of $\bar{V}$ with respect to the $p$-variable solves a linear PDE.

The paper is organized as follows. Section 2 presents the general formulation of singular stochastic target problems and contains the statement of the corresponding dynamic programming equation. The stochastic target problem under controlled probability of success is discussed in Section 3. We first reduce the problem to the setting of the preceeding section in order to obtain directly the corresponding dynamic programming equation inside the domain. A delicate analysis of the boundary conditions is then provided. Section 4 shows how our results reproduce the elegant explicit solution of Föllmer and Leukert [5] in the context of a complete financial market application. The extension to general loss functions is briefly discussed in Section 3.5. Finally, Sections 5 and 6 contain the proofs of the main results.

In all this paper, elements of $\mathbb{R}^{n}, n \geq 1$, are identified to column vectors, the superscript ${ }^{T}$ stands for transposition, - denotes the scalar product on $\mathbb{R}^{n},|\cdot|$ the Euclydean norm, and $\mathbb{M}^{n}$ denotes the set of $n$-dimensional square matrices. We denote by $\mathbb{S}^{n}$ the subset of elements of $\mathbb{M}^{n}$ that are symmetric. For a subset $\mathcal{O}$ of $\mathbb{R}^{n}, n \geq 1$, we denote by $\operatorname{cl}(\mathcal{O})$ its closure, by $\operatorname{int}(\mathcal{O})$ its interior and by $\operatorname{dist}(x, \mathcal{O})$ the Euclidean distance from $x$ to $\mathcal{O}$ with the convention $\operatorname{dist}(x, \emptyset)=\infty$. Finally, we denote by $B_{r}(x)$ the open ball of radius $r>0$ centered at $x \in \mathbb{R}^{n}$. Given a locally 
bounded map $v$ on an open subset $B$ of $\mathbb{R}^{n}$, we define the lower and upper semicontinuous envelopes:

$$
v_{*}(b):=\liminf _{B \ni b^{\prime} \rightarrow b} v\left(b^{\prime}\right), \quad v^{*}(b):=\limsup _{B \ni b^{\prime} \rightarrow b} v\left(b^{\prime}\right) \quad b \in \operatorname{cl}(B) .
$$

In all this paper, inequalities between random variable have to be understood in the a.s. sense.

\section{Singular stochastic target problems}

\subsection{Problem formulation}

Let $T>0$ be the finite time horizon and $W=\left\{W_{t}, 0 \leq t \leq T\right\}$ be a $d$-dimensional Brownian motion defined on a complete probability space $(\Omega, \mathcal{F}, P)$. We denote by $\mathbb{F}=\left\{\mathcal{F}_{t}, 0 \leq t \leq T\right\}$ the $\mathbb{P}$-augmentation of the filtration generated by $W$.

Let $\mathcal{U}_{o}$ be a subset of the collection of progressively measurable processes $\nu$ in $L^{2}([0, T]) \mathbb{P}-$ a.s., with values in a given closed subset $U$ of $\mathbb{R}^{d}$. For $t \in[0, T], z=(x, y) \in \mathbb{R}^{d} \times \mathbb{R}$ and $\nu \in \mathcal{U}_{o}$, we define $Z_{t, z}^{\nu}:=\left(X_{t, x}^{\nu}, Y_{t, z}^{\nu}\right)$ as the $\mathbb{R}^{d} \times \mathbb{R}$-valued solution of the stochastic differential equation:

$$
\begin{aligned}
& d X(r)=\mu\left(X(r), \nu_{r}\right) d r+\sigma\left(X(r), \nu_{r}\right) d W_{r} \\
& d Y(r)=\mu_{Y}\left(Z(r), \nu_{r}\right) d r+\sigma_{Y}\left(Z(r), \nu_{r}\right) \cdot d W_{r}, \quad t \leq r \leq T,
\end{aligned}
$$

satisfying the initial condition $Z(t)=(X(t), Y(t))=(x, y)$. Here,

$$
\begin{aligned}
& \left(\mu_{Y}, \sigma_{Y}\right): \mathbb{R}^{d} \times \mathbb{R} \times U \longrightarrow \mathbb{R} \times \mathbb{R}^{d} \\
& (\mu, \sigma): \mathbb{R}^{d} \times U \longrightarrow \mathbb{R}^{d} \times \mathbb{M}^{d}
\end{aligned}
$$

are locally Lipschitz, and are assumed to satisfy

$$
\left|\mu_{Y}(x, y, u)\right|+|\mu(x, u)|+\left|\sigma_{Y}(x, y, u)\right|+|\sigma(x, u)| \leq K(x, y)(1+|u|),
$$

where $K$ is a locally bounded map.

We denote by $\mathcal{U}$ the subset of elements of $\mathcal{U}_{o}$ for which (2.1) admits a strong solution for all given initial data. We also allow for state constraints and we denote by $\mathbf{X}$ the interior of the support of the controlled process $X$.

Given $u \in U$, we denote by $\mathcal{L}^{u}$ the Dynkin operator associated to the controlled diffusion $X$ :

$$
\mathcal{L}^{u} \varphi(t, x):=\partial_{t} \varphi(t, x)+\mu(x, u) \cdot D \varphi(t, x)+\frac{1}{2} \operatorname{Tr}\left[\sigma \sigma^{\mathrm{T}}(x, u) D^{2} \varphi(t, x)\right]
$$

for a smooth function $\varphi$, where $\partial_{t} \varphi$ stands for the partial derivative with respect to $t, D \varphi$ and $D^{2} \varphi$ denote the gradient vector and the Hessian matrix with respect to the $x$ variable.

Let $G$ be a measurable map from $\mathbb{R}^{d+1}$ to $\mathbb{R}$ such that for every fixed $x$ the function

$$
y \longmapsto G(x, y) \quad \text { is non-decreasing and right-continuous. }
$$


The stochastic target problem is defined by

$$
V(t, x):=\inf \left\{y \in \mathbb{R}: G\left(X_{t, x}^{\nu}(T), Y_{t, x, y}^{\nu}(T)\right) \geq 0 \text { for some } \nu \in \mathcal{U}\right\}
$$

Let us observe that this problem can be formulated equivalently as:

$$
V(t, x)=\inf \left\{y \in \mathbb{R}: Y_{t, x, y}^{\nu}(T) \geq g\left(X_{t, x}^{\nu}(T)\right) \text { for some } \nu \in \mathcal{U}\right\}
$$

where $g$ is the generalized inverse of $G$ at 0 :

$$
g(x):=\inf \{y: G(x, y) \geq 0\} .
$$

When the set $U$ is bounded, it was proved in [9] that the value function $V$ is a discontinuous viscosity solution of:

$$
\sup \left\{\mu_{Y}(x, v(t, x), u)-\mathcal{L}^{u} v(t, x): u \in \mathcal{N}_{0}(x, v(t, x), D v(t, x))\right\}=0,
$$

where

$$
\mathcal{N}_{0}(x, y, q):=\left\{u \in U: N^{u}(x, y, q)=0\right\} \text { and } N^{u}(x, y, q):=\sigma_{Y}(x, y, u)-\sigma(x, u)^{\mathrm{T}} q
$$

Since $\mathcal{N}_{0}(x, y, q)$ may be empty, we shall use the standard convention $\sup \emptyset=-\infty$ all over this paper.

The chief goal of this section is to provide an extension of this result to the case where $U$ is unbounded.

\subsection{The dynamic programming equation}

Because the control set $U$ is not necessarily bounded, we need to introduce the relaxed semilimits:

$$
F^{*}(\Theta):=\limsup _{\varepsilon \backslash 0, \Theta^{\prime} \rightarrow \Theta} F_{\varepsilon}\left(\Theta^{\prime}\right) \quad \text { and } \quad F_{*}(\Theta):=\liminf _{\varepsilon \backslash 0, \Theta^{\prime} \rightarrow \Theta} F_{\varepsilon}\left(\Theta^{\prime}\right)
$$

where, for $\Theta=(x, y, q, A) \in \mathbb{R}^{d} \times \mathbb{R} \times \mathbb{R}^{d} \times \mathbb{S}^{d}$ and $\varepsilon \geq 0$,

$$
F_{\varepsilon}(\Theta):=\sup \left\{\mu_{Y}(x, y, u)-\mu(y, u) \cdot q-\frac{1}{2} \operatorname{Tr}\left[\sigma \sigma^{\mathrm{T}}(x, u) A\right]: u \in \mathcal{N}_{\varepsilon}(x, y, q)\right\}
$$

and

$$
\mathcal{N}_{\varepsilon}(x, y, q):=\left\{u \in U:\left|N^{u}(x, y, q)\right| \leq \varepsilon\right\}, \quad \varepsilon \geq 0
$$

Observe that $\left(\mathcal{N}_{\varepsilon}\right)_{\varepsilon \geq 0}$ is non-decreasing so that

$$
F_{*}(\Theta):=\liminf _{\Theta^{\prime} \rightarrow \Theta} F_{0}\left(\Theta^{\prime}\right) .
$$

For ease of notations, we shall often simply write $F v(t, x)$ in place of $F(x, v(t, x), D v(t, x)$, $\left.D^{2} v(t, x)\right)$. We shall similarly use the notations $F^{*} v$ and $F_{*} v$. 
Our first main result is the derivation of the dynamic programming equation corresponding to the stochastic target problem in the present context of possibly unbounded controls. This is an extension of [9] and [10] where the set $U$ was assumed to be bounded, see also [2] for the case of jump diffusions. This extension is crucial for our analysis of the stochastic target problem under controlled probability, and under controlled loss. The following continuity assumption is needed in order to prove the subsolution property. Note that this version is slightly weaker than Assumption 4.1 in $[10]$.

Assumption 2.1 (Continuity of $\mathcal{N}_{0}(x, y, q)$ ) Let $B$ be a subset of $\mathbf{X} \times \mathbb{R} \times \mathbb{R}^{d}$ such that $\mathcal{N}_{0} \neq \emptyset$ on $B$. Then, for every $\varepsilon>0,\left(x_{0}, y_{0}, q_{0}\right) \in \operatorname{int}(B)$, and $u_{0} \in \mathcal{N}_{0}\left(x_{0}, y_{0}, q_{0}\right)$, there exists an open neighborhood $B^{\prime}$ of $\left(x_{0}, y_{0}, q_{0}\right)$ and a locally Lipschitz map $\hat{\nu}$ defined on $B^{\prime}$ such that $\mid \hat{\nu}\left(x_{0}, y_{0}, q_{0}\right)-$ $u_{0} \mid \leq \varepsilon$ and $\hat{\nu}(x, y, q) \in \mathcal{N}_{0}(x, y, q)$ on $B^{\prime}$.

Throughout this paper, we shall always assume the following

Standing Assumption $V$ is locally bounded,

so that the semilimits $V_{*}$ and $V^{*}$ are finite. Our first main result characterizes $V$ as a discontinuous viscosity solution of (2.7) in the following sense.

Theorem 2.1 The function $V_{*}$ is a viscosity supersolution of

$$
-\partial_{t} V_{*}+F^{*} V_{*} \geq 0 \quad \text { on } \quad[0, T) \times \mathbf{X} .
$$

If in addition Assumption 2.1 holds, then $V^{*}$ is a viscosity subsolution of

$$
-\partial_{t} V^{*}+F_{*} V^{*} \leq 0 \quad \text { on } \quad[0, T) \times \mathbf{X} .
$$

The proof of this result is reported in Section 5. In particular, the supersolution property is proved by a new approach, and avoids delicate passages to limits that appear in [10].

Let us now introduce the set-valued map

$$
\mathbf{N}(x, y, q):=\left\{r \in \mathbb{R}^{d}: r=N^{u}(x, y, q) \text { for some } u \in U\right\},
$$

together with the signed distance function from its complement set $\mathbf{N}^{c}$ to the origin:

$$
\delta:=\operatorname{dist}\left(0, \mathbf{N}^{c}\right)-\operatorname{dist}(0, \mathbf{N}),
$$

where we recall that dist stands for the (unsigned) Euclidean distance. Then,

$$
0 \in \operatorname{int}(\mathbf{N}(x, y, q)) \quad \text { iff } \quad \delta(x, y, q)>0 .
$$

The upper and lower-semicontinuous envelopes of $\delta$ are respectively denoted by $\delta^{*}$ and $\delta_{*}$, and we will abuse notation by writing $\delta_{*} v(x)=\delta_{*}(x, v(x), D v(x))$ and $\delta^{*} v(x)=\delta^{*}(x, v(x), D v(x))$. 
Remark 2.1 From the convention $\sup \emptyset=-\infty$ and the supersolution property (2.10) in Theorem 2.1 , it follows that

$$
\delta^{*} V_{*} \geq 0 \quad \text { on } \quad[0, T) \times \mathbb{R}^{d}
$$

in the viscosity sense. Then, if $\mathbf{N}^{c} \neq \emptyset$, this means that $V$ is subject to a gradient constraint.

Remark 2.2 Let us check that Theorem 2.1 reduces to the viscosity property of [9] in their setting. Assume that for every $(x, y, q)$ and $r \in \mathbb{R}^{d}$, there is a unique solution $\hat{u}(x, y, q, r)$ to the equation $N^{u}(x, y, q)=r$, i.e.

$$
N^{u}(x, y, q)=r \quad \text { iff } \quad u=\hat{u}(x, y, q, r)
$$

Assume further that $\hat{u}$ is locally Lipschitz continuous, so that Assumption 2.1 trivially holds. For ease of notations, we set $\hat{u}_{0}(x, y, q):=\hat{u}(x, y, q, 0)$. For a bounded set of controls $U$, it follows that for any smooth function $\varphi: F^{*} \varphi(t, x) \geq 0$ implies that

$$
\hat{u}_{0}(x, \varphi(t, x), D \varphi(t, x)) \in U \quad \text { and } \quad-\partial_{t} \varphi(t, x)+F_{0} \varphi(t, x) \geq 0
$$

where the operator $F_{0}$ reduces in the present context to

$$
F_{0} \varphi=\mu_{Y}\left(., \varphi, \hat{u}_{0}\right)-\mu\left(\varphi, \hat{u}_{0}\right) \cdot D \varphi-\frac{1}{2} \operatorname{Tr}\left[\sigma \sigma^{\mathrm{T}}\left(., \hat{u}_{0}\right) D^{2} \varphi\right] .
$$

Similarly, $F_{*} \varphi(t, x) \leq 0$ implies that

$$
\text { either } \hat{u}_{0}(x, \varphi(t, x), D \varphi(t, x)) \notin \operatorname{int}(U) \quad \text { or } \quad-\partial_{t} \varphi(t, x)+F_{0} \varphi(t, x) \leq 0 \text {. }
$$

Notice that (2.17)-(2.18) correspond to the PDE derived in [9].

We next discuss the boundary condition on $\{T\} \times \mathbf{X}$. By the definition of the stochastic target problem, we have

$$
V(T, x)=g(x) \text { for every } \quad x \in \mathbb{R}^{d}
$$

However, the possible discontinuities of $V$ might imply that the limits $V_{*}(T,$.$) and V^{*}(T,$.$) do$ not agree with this "natural" boundary condition. The following result states that the constraint discussed in Remark 2.1 propagates up to the boundary. Again, this phenomenon was already noticed in [9], among others. Here, the main difficulty is due to the unboundedness of the set $U$.

Theorem 2.2 The function $x \in \mathbf{X} \mapsto V_{*}(T, x)$ is a viscosity supersolution of

$$
\min \left\{\left(V_{*}(T, \cdot)-g_{*}\right) \mathbf{1}_{\left\{F^{*} V_{*}(T, \cdot)<\infty\right\}}, \delta^{*} V_{*}(T, \cdot)\right\} \geq 0 \quad \text { on } \mathbf{X},
$$

and, under Assumption 2.1, $x \in \mathbf{X} \mapsto V^{*}(T, x)$ is a viscosity subsolution of

$$
\min \left\{V^{*}(T, \cdot)-g^{*}, \delta_{*} V^{*}(T, \cdot)\right\} \leq 0 \quad \text { on } \mathbf{X} \text {. }
$$


Remark 2.3 Note that $\delta(x, y, q) \leq 0$ whenever $\operatorname{int}(\mathbf{N}(x, y, q))=\emptyset$, so that the subsolution property carries no information. This is the case when the control set $U$ has empty interior as in the context of a stochastic volatility model. A specific analysis is needed in such cases, see [11] and [7].

Remark 2.4 In the context of Remark 2.2, observe that

- $\delta^{*} \varphi(x) \geq 0$ implies that $\hat{u}_{0}(x, \varphi(x), D \varphi(x)) \in U$, and $F^{*} \varphi(x)<\infty$ is always satisfied,

- $\delta_{*} \varphi(x)>0$ implies that $\hat{u}_{0}(x, \varphi(x), D \varphi(x)) \in \operatorname{int}(U)$.

Hence, for a convex set $U$ with non-empty interior, we recover the boundary condition of [9].

When $\mathbf{X}=\mathbb{R}^{d}$ (and under suitable conditions) a comparison result of viscosity supersolutions

of (2.10)-(2.19) and subsolutions of (2.11)-(2.20) can be proved in certain classes of functions. We do not persue this issue any further, and we instead refer to [3] for some general results in this direction and to [1] for a comparison result in a similar setting. We recall that the main concern of this paper is the analysis of the stochastic target problem under controlled probability or, more generally, controlled loss. However, we shall assume that such a comparison result holds in order to establish the boundary conditions for the problem of stochastic target under controlled probability and/or loss of the subsequent section.

Assumption 2.2 There is a class of functions $\mathcal{C}$ containing all non-negative functions dominated by $V^{*}$ such that, for every

- $v_{1} \in \mathcal{C}$, lower-semicontinuous viscosity supersolution of $(2.10)-(2.19)$ on $[0, T] \times \mathbf{X}$,

- $v_{2} \in \mathcal{C}$, upper-semicontinuous viscosity subsolution of $(2.11)-(2.20)$ on $[0, T] \times \mathbf{X}$, we have $v_{1} \geq v_{2}$.

Note that, when the process $X$ is subject to state constraints, the boundary conditions on $[0, T] \times \partial \mathbf{X}$ have to be specified. We deliberately avoid this issue for sake of simplicity. However, in our subsequent analysis, it will appear from the very nature of the problem, and we will deal with a special type of state constraints, see Subsection 3.3 .

\section{Target reachability with controlled probability of success}

In this section, we extend the model presented above to the case where the target has to be reached only with a given probability $p$ :

$$
\bar{V}(t, x, p):=\inf \left\{y \in \mathbb{R}_{+}: \mathbb{P}\left[G\left(X_{t, x}^{\nu}(T), Y_{t, x, y}^{\nu}(T)\right) \geq 0\right] \geq p \text { for some } \nu \in \mathcal{U}\right\} .
$$

In order to avoid degenerate results, we restrict the analysis to the case where the $Y$ process takes non-negative values, by simply imposing the following conditions on the coefficients driving its dynamics:

$$
\mu_{Y}(x, 0, u) \geq 0 \text { and } \sigma_{Y}(x, 0, u)=0 \quad \text { for all }(x, u) \in \mathbf{X} \times U .
$$

Note that the above definition implies that

$$
0=\bar{V}(\cdot, 0) \leq \bar{V} \leq \bar{V}(\cdot, 1)=V .
$$




\subsection{Problem reduction}

Our first objective is to convert this problem into the class of (standard) stochastic target problems, so that the dynamic programming equation for the target reachability problem with controlled probability can be deduced directly from Theorem 2.1. To do this, we introduce an additional controlled state variable valued in $[0,1]$ and defined by:

$$
P_{t, p}^{\alpha}=p, \quad d P_{t, p}^{\alpha}(s)=P_{t, p}^{\alpha}(s)\left(1-P_{t, p}^{\alpha}(s)\right) \alpha_{s} \cdot d W_{s}, \quad s \in[t, T]
$$

where the additional control $\alpha$ is an $\mathbb{F}$-progressively measurable $\mathbb{R}^{d}$-valued $\mathbb{P}-$ a.s. square integrable process. We next set $\bar{X}:=(X, P), \overline{\mathbf{X}}:=\mathbf{X} \times(0,1), \bar{U}:=U \times \mathbb{R}^{d}$, denote by $\overline{\mathcal{U}}$ the corresponding set of admissible controls, and set

$$
\bar{G}(\bar{x}, y):=\mathbf{1}_{\{G(x, y) \geq 0\}}-p \quad \text { for } \quad y \in \mathbb{R}, \bar{x}:=(x, p) \in \operatorname{cl}(\overline{\mathbf{X}}) .
$$

Proposition 3.1 For all $t \in[0, T]$ and $\bar{x}=(x, p) \in \overline{\mathbf{X}}$, we have

$$
\bar{V}(t, \bar{x})=\inf \left\{y \in \mathbb{R}_{+}: \bar{G}\left(\bar{X}_{t, \bar{x}}^{\bar{\nu}}(T), Y_{t, x, y}^{\nu}(T)\right) \geq 0 \text { for some } \bar{\nu}=(\nu, \alpha) \in \overline{\mathcal{U}}\right\} .
$$

Proof. We denote by $v(t, x, p)$ the right-hand side of (3.5). For $y>\bar{V}(t, x, p)$, we can find $\nu \in \mathcal{U}$ such that $\mathbb{P}\left[G\left(X_{t, x}^{\nu}(T), Y_{t, x, y}^{\nu}(T)\right) \geq 0\right] \geq p$. By the stochastic integral representation theorem, there exists an $\mathbb{F}$-progressively measurable $\mathbb{P}$ - a.s.-square integrable process $\phi$ such that

$$
P(T):=p+\mathbf{1}_{\left\{G\left(X_{t, x}^{\nu}(T), Y_{t, x, y}^{\nu}(T)\right) \geq 0\right\}}-\mathbb{P}\left[G\left(X_{t, x}^{\nu}(T), Y_{t, x, y}^{\nu}(T)\right) \geq 0\right]=p+\int_{t}^{T} \phi_{s} \cdot d W_{s} .
$$

Since $P(T) \in[0,1]$, it is clear that $P(T)=P_{t, p}^{\alpha}(T)$ for some progressively measurable $\mathbb{P}-$ a.s.square integrable process $\alpha$. Hence $\bar{\nu}:=(\nu, \alpha) \in \overline{\mathcal{U}}$. Observing from the above equality that $\bar{G}\left(\bar{X}_{t, \bar{x}}^{\bar{\nu}}(T), Y_{t, x, y}^{\nu}(T)\right)=\mathbb{P}\left[G\left(X_{t, x}^{\nu}(T), Y_{t, x, y}^{\nu}(T)\right) \geq 0\right]-p \geq 0$, we deduce from the arbitrariness of $y>\bar{V}(t, x, p)$ that $\bar{V}(t, x, p) \geq v(t, x, p)$.

Conversely, for $y>v(t, x, p)$, we have $\bar{G}\left(\bar{X}_{t, \bar{x}}^{\bar{\nu}}(T), Y_{t, x, y}^{\nu}(T)\right) \geq 0$ for some $\bar{\nu}=(\nu, \alpha) \in \overline{\mathcal{U}}$. Since $P_{t, p}^{\alpha}$ is a martingale (as a bounded local martingale), it follows that

$$
\mathbb{P}\left[G\left(X_{t, x}^{\nu}(T), Y_{t, x, y}^{\nu}(T)\right) \geq 0\right]=\mathbb{E}\left[\mathbf{1}_{\left\{G\left(X_{t, x}^{\nu}(T), Y_{t, x, y}^{\nu}(T)\right) \geq 0\right\}}\right] \geq \mathbb{E}\left[P_{t, p}^{\alpha}(T)\right]=p,
$$

which concludes the proof of (3.5).

\subsection{The dynamic programming equation}

The above reduction of the problem $\bar{V}$ to a stochastic target problem allows to apply the general results of the previous section. For $\bar{u}=(u, \alpha) \in \bar{U}$, set

$$
\bar{\mu}(\bar{x}, \bar{u}):=\left(\begin{array}{c}
\mu(x, u) \\
0
\end{array}\right), \quad \bar{\sigma}(\bar{x}, \bar{u}):=\left(\begin{array}{c}
\sigma(x, u) \\
\alpha^{\mathrm{T}}
\end{array}\right),
$$


where for presentation simplicity, we omit $p(1-p)$ in the diffusion of the state variable $P$. Note that for $p \in(0,1)$, this only corresponds to a change of variable in the PDEs below. We also introduce for $(y, q, A) \in \mathbb{R} \times \mathbb{R}^{d+1} \times \mathbb{S}^{d+1}$ and still $\bar{u}=(u, \alpha) \in \bar{U}$,

$$
\begin{gathered}
\bar{N}^{\bar{u}}(\bar{x}, y, q):=\sigma_{Y}(x, y, u)-\bar{\sigma}(\bar{x}, \bar{u})^{\mathrm{T}} q=N^{u}\left(x, y, q_{x}\right)-q_{p} \alpha \quad \text { for } \quad q=\left(q_{x}, q_{p}\right) \in \mathbb{R}^{d} \times \mathbb{R}, \\
\overline{\mathcal{N}}_{\varepsilon}(\bar{x}, y, q):=\left\{\bar{u} \in \bar{U}:\left|\bar{N}^{\bar{u}}(\bar{x}, y, q)\right| \leq \varepsilon\right\}, \quad \varepsilon>0, \\
\bar{F}_{\varepsilon}(\bar{x}, y, q, A):=\sup _{(u, \alpha) \in \overline{\mathcal{N}}_{\varepsilon}(\bar{x}, y, q)}\left\{\mu_{Y}(x, y, u)-\bar{\mu}(\bar{x}, u, \alpha) \cdot q-\frac{1}{2} \operatorname{Tr}\left[\bar{\sigma} \bar{\sigma}^{\mathrm{T}}(\bar{x}, u, \alpha) A\right]\right\},
\end{gathered}
$$

and

$$
\overline{\mathbf{N}}(\bar{x}, y, q):=\left\{N^{\bar{u}}(\bar{x}, y, q): \bar{u} \in \bar{U}\right\}, \quad \bar{\delta}:=\operatorname{dist}\left(0, \overline{\mathbf{N}}^{c}\right)-\operatorname{dist}(0, \overline{\mathbf{N}}) .
$$

The operators $\bar{F}^{*}$ and $\bar{F}_{*}$ are constructed from $\bar{F}_{\varepsilon}$ exactly as $F^{*}$ and $F_{*}$ are defined from $F_{\varepsilon}$. Finally, we define the function

$$
\bar{g}(\bar{x}):=\inf \{y \geq 0: \bar{G}(\bar{x}, y) \geq 0\}, \bar{x}=(x, p) \in \overline{\mathbf{X}} \times[0,1],
$$

which is related to $g$ by

$$
\bar{g}(x, p)=g(x) \mathbf{1}_{p>0} \quad \text { for } \quad x \in \mathbf{X} \text { and } p \in[0,1] .
$$

As an almost direct consequence of Theorem 2.1 and (3.5), we obtain the viscosity property of $\bar{V}$ under the following assumption which is the analog of Assumption 2.1 for the augmented control system $\bar{X}$.

Assumption 3.1 (Continuity of $\overline{\mathcal{N}}_{0}(x, p, y, q)$ ) Let $B$ be a subset of $\mathbf{X} \times[0,1] \times \mathbb{R} \times \mathbb{R}^{d+1}$ such that $\overline{\mathcal{N}}_{0} \neq \emptyset$ on $B$. Then, for every $\varepsilon>0,\left(x_{0}, p_{0}, y_{0}, q_{0}\right) \in \operatorname{int}(B)$, and $\bar{u}_{0} \in \overline{\mathcal{N}}_{0}\left(x_{0}, p_{0}, y_{0}, q_{0}\right)$, there exists an open neighborhood $B^{\prime}$ of $\left(x_{0}, p_{0}, y_{0}, q_{0}\right)$ and a locally Lipschitz map $\hat{\nu}$ defined on $B^{\prime}$ such that $\left|\hat{\nu}\left(x_{0}, p_{0}, y_{0}, q_{0}\right)-\bar{u}_{0}\right| \leq \varepsilon$ and $\hat{\nu}(x, p, y, q) \in \overline{\mathcal{N}}_{0}(x, p, y, q)$ on $B^{\prime}$.

Corollary 3.1 The function $\bar{V}_{*}$ is a viscosity supersolution of

$$
-\partial_{t} \bar{V}_{*}+\bar{F}^{*} \bar{V}_{*} \geq 0 \quad \text { on } \quad[0, T) \times \overline{\mathbf{X}} .
$$

Under the additional Assumption 3.1, $\bar{V}^{*}$ is a viscosity subsolution of

$$
\min \left\{\bar{V}^{*},-\partial_{t} \bar{V}^{*}+\bar{F}_{*} \bar{V}^{*}\right\} \leq 0 \quad \text { on }[0, T) \times \overline{\mathbf{X}} .
$$

Proof. The supersolution property is a direct consequence of Theorem 2.1. The subsolution property is obtained similarly. The first term accounts for the non-negativity constraint on the state process $Y$ in the arguments of the proof in Section 5.3 below.

Remark 3.1 Clearly, Assumption 3.1 holds true whenever the function $\hat{u}$ introduced in Remark 2.2 is well defined and locally Lipschitz continuous. 


\subsection{Boundary conditions and state constraint}

The above result relies on converting $\bar{V}$ into a (singular) stochastic target problem. This was achieved by introducing the new state variable $p$. Because this variable is constrained in $[0,1]$, we need to specify the boundary conditions at the endpoints 0 and 1. By definition of the stochastic target problem with controlled probability, we have

$$
\bar{V}(\cdot, 1)=V \text { and } \bar{V}(\cdot, 0)=0 .
$$

Also, since $G$ is non-decreasing in $y$, we know that $\bar{V}$ is non-decreasing in $p$. Hence

$$
0 \leq \bar{V}_{*}(\cdot, 0) \leq \bar{V}^{*}(\cdot, 1) \leq V^{*}
$$

and one can naturally expect that $\bar{V}_{*}(\cdot, 0)=0$ and $\bar{V}^{*}(\cdot, 1)=V^{*}$. However, the function $V$ may have discontinuities at $p=0$ or $p=1$ and, in general, the boundary conditions have to be stated in a weak form. To obtain a characterization of $V$ on these boundaries, we shall appeal to the following additional assumptions.

Assumption 3.2 For all $(x, y, q) \in \mathbf{X} \times(0, \infty) \times \mathbb{R}^{d}$, we have $\mathcal{N}_{0}(x, y, q) \subsetneq U$.

Assumption 3.3 For all compact subset $A$ of $\mathbb{R}^{d} \times \mathbb{R} \times \mathbb{R}^{d} \times \mathbb{S}^{d}$, there exists $C>0$ such that $F_{\varepsilon}(\Theta) \leq C\left(1+\varepsilon^{2}\right)$ for all $\varepsilon \geq 0$ and $\Theta \in A$.

Remark 3.2 Assumption 3.2 is natural and allows to avoid degenerate cases that would have to be discussed separately. It will be used only to derive the boundary condition at $p=0$. Assumption 3.3 is more of technical nature and will be only used to discuss the boundary condition at $p=1$.

The main result of this section shows that the natural boundary conditions (3.6) indeed holds true, whenever the comparison principle of Assumption 2.2 holds and under the above additional assumptions.

Theorem 3.1 Assume that the function $\sup _{u \in U}|\sigma(\cdot, u)|$ is locally bounded on $\mathbf{X}$ and that Assumption 3.1 holds true.

(i) Under Assumption 3.2, we have $\bar{V}^{*}(\cdot, 0)=0$ on $[0, T) \times \mathbf{X}$ and $\bar{V}_{*}(\cdot, 0)=0$ on $[0, T] \times \mathbf{X}$.

(ii) Under Assumption 3.3, $\bar{V}^{*}(\cdot, 1)$ is a viscosity supersolution of $(2.10)-(2.19)$ on $[0, T] \times \mathbf{X}$. In particular, if in addition the comparison Assumption 2.2 is satisfied, then $\bar{V}^{*}(\cdot, 1)=\bar{V}_{*}(\cdot, 1)=$ $V_{*}=V^{*}$ on $[0, T] \times \mathbf{X}$.

The proof is reported in Section 6 .

\subsection{On the terminal condition}

The boundary condition at $T$ for $\bar{V}_{*}$ and $\bar{V}^{*}$ can be easily derived from the characterization of Theorem 2.2. 
Corollary 3.2 The function $\bar{x} \in \overline{\mathbf{X}} \mapsto \bar{V}_{*}(T, \bar{x})$ is a viscosity supersolution of

$$
\min \left\{\left(\bar{V}_{*}(T, \cdot)-\bar{g}_{*}\right) \mathbf{1}_{\left\{\bar{F}^{*} \bar{V}_{*}(T, \cdot)<\infty\right\}}, \bar{\delta}^{*} \bar{V}_{*}(T, \cdot)\right\} \geq 0 \quad \text { on } \overline{\mathbf{X}} .
$$

If in addition Assumption 3.1 holds, then $\bar{x} \in \overline{\mathbf{X}} \mapsto \bar{V}^{*}(T, \bar{x})$ is a viscosity subsolution of

$$
\min \left\{\bar{V}^{*}(T, \cdot)-\bar{g}^{*}, \bar{\delta}_{*} \bar{V}^{*}(T, \cdot)\right\} \leq 0 \quad \text { on } \overline{\mathbf{X}} .
$$

Note however that $\bar{g}^{*}=g^{*}$ so that the discontinuity in the $p$ variable of the boundary condition on $\bar{V}^{*}$ is not apparent in the above formulation. Moreover the condition $\bar{F}^{*} \bar{V}_{*}(T, \cdot)<\infty$ may not be satisfied because of the unboundeness of the control $\alpha$ that appears in the definition of $\bar{F}$. It follows that the above boundary condition may be useless in most examples.

In the rest of this section, we provide conditions under which a more precise boundary condition can be specified. These assumptions will be satisfied in our example of application, see Section 4 below.

Proposition 3.2 (i) Assume that $g$ is continuous and that for all sequence $\left(t_{n}, x_{n}, y_{n}, p_{n}, \nu_{n}\right)_{n}$ of $[0, T) \times \mathbf{X} \times \mathbb{R}_{+} \times[0,1] \times \mathcal{U}$ such that $\left(t_{n}, x_{n}, y_{n}, p_{n}\right) \rightarrow(T, x, y, p) \in\{T\} \times \mathbf{X} \times \mathbb{R}_{+} \times[0,1]$, there exists a $\mathbb{P}$-absolutely continuous probability measure $\mathbb{Q}$ such that

$$
\limsup _{n \rightarrow \infty} \mathbb{E}^{\mathbb{Q}}\left[Y_{t_{n}, x_{n}, y_{n}}^{\nu_{n}}(T)\right] \leq y \quad \text { and } \quad \limsup _{n \rightarrow \infty} \mathbb{E}^{\mathbb{Q}}\left[\left|g\left(X_{t_{n}, x_{n}}^{\nu_{n}}(T)\right)-g(x)\right|\right]=0 .
$$

Then, $\bar{V}_{*}(T, x, p) \geq p g(x)$ for all $(x, p) \in \mathbf{X} \times[0,1]$.

(ii) Let the conditions of Theorem 3.1 hold true and assume that $\bar{V}^{*}$ is convex in its $p$ variable and that $V^{*}(T, \cdot) \leq g$. Then, $\bar{V}^{*}(T, x, p) \leq p g(x)$ for all $(x, p) \in \mathbf{X} \times[0,1]$.

Proof. It follows from Theorem 3.1 and the convexity property of $\bar{V}^{*}$ that $\bar{V}^{*}(t, x, p) \leq p V^{*}(t, x)$ for all $(t, x, p) \in[0, T) \times \mathbf{X} \times[0,1]$. Since $V^{*}(T, \cdot) \leq g$ by assumption, we deduce that $\bar{V}^{*}(T, \cdot, p) \leq p g$ by considering a sequence $\left(t_{n}, x_{n}, p_{n}\right)_{n}$ in $[0, T) \times \mathbf{X} \times(0,1)$ such that $\left(t_{n}, x_{n}, p_{n}\right) \rightarrow(T, x, p)$ and $\bar{V}^{*}\left(t_{n}, x_{n}, p_{n}\right) \rightarrow \bar{V}^{*}(T, x, p)$. On the other hand, given a sequence $\left(t_{n}, x_{n}, p_{n}\right)_{n}$ in $[0, T) \times \mathbf{X} \times(0,1)$ such that $\left(t_{n}, x_{n}, p_{n}\right) \rightarrow(T, x, p)$ and $\bar{V}\left(t_{n}, x_{n}, p_{n}\right) \rightarrow \bar{V}_{*}(T, x, p)$, we can find $\left(\nu_{n}, \alpha_{n}\right) \in \overline{\mathcal{U}}$ such that

$$
\mathbf{1}_{\left\{Y_{t_{n}, x_{n}, y_{n}}^{\nu_{n}}(T)-g\left(X_{t_{n}, x_{n}}^{\nu_{n}}(T)\right) \geq 0\right\}} \geq P_{t_{n}, p_{n}}^{\alpha_{n}}(T)
$$

where $y_{n}:=\bar{V}\left(t_{n}, x_{n}, p_{n}\right)+1 / n \rightarrow \bar{V}_{*}(T, x, p)$. This implies that

$$
Y_{t_{n}, x_{n}, y_{n}}^{\nu_{n}}(T) \geq P_{t_{n}, p_{n}}^{\alpha_{n}}(T) g\left(X_{t_{n}, x_{n}}^{\nu_{n}}(T)\right)
$$

and, since $P_{t_{n}, p_{n}}^{\alpha_{n}}(T)$ is bounded by 1 ,

$$
Y_{t_{n}, x_{n}, y_{n}}^{\nu_{n}}(T) \geq P_{t_{n}, p_{n}}^{\alpha_{n}}(T) g(x)-\left|g\left(X_{t_{n}, x_{n}}^{\nu_{n}}(T)\right)-g(x)\right|
$$

Taking the expectation under $\mathbb{Q}$ and recalling that $P_{t_{n}, p_{n}}^{\alpha_{n}}$ is a bounded martingale, we get

$$
\mathbb{E}^{\mathbb{Q}}\left[Y_{t_{n}, x_{n}, y_{n}}^{\nu_{n}}(T)\right] \geq p_{n} g(x)-\mathbb{E}^{\mathbb{Q}}\left[\left|g\left(X_{t_{n}, x_{n}}^{\nu_{n}}(T)\right)-g(x)\right|\right] \text {. }
$$


Passing to the limit and using (3.10) leads to $\bar{V}_{*}(T, x, p) \geq p g(x)$.

The conditions of Proposition 3.2 are easily satisfied if the coefficients of $X^{\nu}$ and $Y^{\nu}$ are Lipschitz continuous uniformly in the control variable. The condition on $Y^{\nu}$ also typically holds if, after a suitable change of measure, the control appears in its dynamics only through the Itô integral. This is typically the case in finance where $Y^{\nu}$ plays the role of the wealth process, see the example of Section 4 below.

We now provide conditions ensuring the convexity of $\bar{V}^{*}$ in its $p$ variable.

Proposition 3.3 Assume that $\bar{V}^{*}>0$ on $[0, T) \times \mathbf{X} \times(0,1)$ and that $U=\mathbb{R}^{d}$. Assume further that the function $\hat{u}$ defined in (2.16) is locally Lipschitz continuous, that $\hat{u}(x, y, q, \cdot)$ is Lipschitz on $\mathbb{R}^{d}$ for all $(x, y, q) \in \mathbf{X} \times \mathbb{R} \times \mathbb{R}^{d}$, and that for each compact set $\Theta \subset \mathbf{X} \times \mathbb{R}_{+}$there exists $C>0$ and $\varepsilon \in(0,2]$ such that

$$
\left|\mu_{Y}(x, y, u)\right|+|\mu(x, u)|+|\sigma(x, u)|^{2} \leq C\left(1+|u|^{2-\varepsilon}\right) \quad \text { for all }(x, y, u) \in \Theta \times U .
$$

Then, $\bar{V}^{*}(t, x, p)$ is convex in $p$.

Proof. Since $U=\mathbb{R}^{d}$, it follows from the same argument as in Remark 2.2 that the condition of Corollary 3.1 is satisfied. Since $\bar{V}^{*}>0$, this implies that $\bar{V}^{*}$ is a viscosity subsolution of $-\partial_{t} \bar{V}^{*}+\bar{F}_{*} \bar{V}^{*} \leq 0$ on $[0, T) \times \overline{\mathbf{X}}$. Let $\varphi$ be a smooth function and $(t, x, p)$ be a local maximizer of $\bar{V}^{*}-\varphi$ on $[0, T) \times \overline{\mathbf{X}}$. Note that, by definition of $\hat{u}$ and the assumption $U=\mathbb{R}^{d}$,

$$
\overline{\mathcal{N}}_{0}\left(x, y,\left(q_{x}, q_{p}\right)\right)=\left\{\left(\hat{u}\left(x, y, q_{x}, \alpha q_{p}\right), \alpha\right), \alpha \in \mathbb{R}\right\}
$$

In view of the growth condition (3.11) and the Lipschitz continuity assumption on $\hat{u}$, this implies that there exists $C>0$ and $\varepsilon \in(0,2]$ such that, for all $\alpha \in \mathbb{R}$,

$$
-\frac{1}{2}|\alpha|^{2} D_{p p} \varphi(t, x, p) \leq C\left(1+|\alpha|^{2-\varepsilon}\right)
$$

This implies that $D_{p p} \varphi(t, x, p) \geq 0$. The convexity then follows from the same arguments as in [4, Proposition 5.2].

\subsection{Extension to target reachability with controlled expected loss}

We now briefly explain how to extend the key-idea of Proposition 3.1 to the target reachability problem with controlled expected loss.

Let $\ell: \mathbb{R} \longrightarrow \mathbb{R}$ be a non-decreasing function, and denote by

$$
L:=\overline{\operatorname{conv}}\left(\ell \circ G\left(\mathbf{X} \times \mathbb{R}_{+}\right)\right)
$$

the closed convex hull of the image of $\ell \circ G$. For $p \in L$, we define the target reachability problem with controlled loss:

$$
\bar{V}^{\ell}(t, x, p):=\inf \left\{y \in \mathbb{R}_{+}: \mathbb{E}\left[\ell \circ G\left(X_{t, x}^{\nu}(T), Y_{t, x, y}^{\nu}(T)\right)\right] \geq p \text { for some } \nu \in \mathcal{U}\right\} .
$$


Observe that for $\ell(r)=\mathbf{1}_{r \geq 0}$ we recover the target reachability problem with controlled probability. As in the previous section, we introduce an additional controlled state variable valued in $L$ defined by:

$$
P_{t, p}^{\alpha}=p, \quad d P_{t, p}^{\alpha}(u)=\alpha_{u} \cdot d W_{u}, \quad u \in[t, T]
$$

where the additional control $\alpha$ is an $\mathbb{F}$-progressively measurable real valued process such that $P^{\alpha}$ is a square integrable martingale taking values in $L, \mathbb{P}-$ a.s. We next denote by $\bar{X}:=(X, P)$, $\bar{U}=U \times \mathbb{R}^{d}, \overline{\mathcal{U}}$ the corresponding set of admissible controls, and

$$
\bar{G}^{\ell}(\bar{x}, y):=\ell \circ G(x, y)-p, y \in \mathbb{R}_{+}, \bar{x}=(x, p) \in \operatorname{cl}(\overline{\mathbf{X}}) \quad \text { where } \quad \overline{\mathbf{X}}=\mathbf{X} \times L .
$$

If $\ell \circ G\left(X_{t, x}^{\nu}(T), Y_{t, x, y}^{\nu}(T)\right)$ is square integrable for all initial conditions and controls $\nu \in \mathcal{U}$, we can then follow the arguments used in the proof of Proposition 3.1 and relate $\bar{V}^{\ell}$ to a stochastic target problem with unbounded controls:

$$
\bar{V}^{\ell}(t, \bar{x})=\inf \left\{y \in \mathbb{R}_{+}: \bar{G}^{\ell}\left(\bar{X}_{t, \bar{x}}^{\bar{\nu}}(T), Y_{t, x, y}^{\nu}(T)\right) \geq 0 \text { for some } \bar{\nu}=(\nu, \alpha) \in \overline{\mathcal{U}}\right\} .
$$

This allows to provide a PDE characterization of $\bar{V}^{\ell}$ in the spirit of the one obtained for $\bar{V}$ above. The adaptation of the previous arguments to this context is obvious.

\section{Application to the quantile hedging problem}

In this section, we specialize the discussion to the quantile hedging problem of Föllmer and Leukert [5]. We first assume that the state space of the process $X$ is $\mathbf{X}:=(0, \infty)^{d}$ and that it is not affected by the control:

$$
\mu(x, u)=\mu(x) \text { and } \sigma(x, u)=\sigma(x) \text { are independent of } u,
$$

where $\mu$ and $\sigma$ are Lipschitz continuous. In order to avoid arbitrage, we also assume that $\sigma$ is invertible and that

$$
\sup _{x \in(0, \infty)^{d}}|\lambda(x)|<\infty \quad \text { where } \quad \lambda:=\sigma^{-1} \mu .
$$

The coefficients of the controlled process $Y$ are given by:

$$
\mu_{Y}(x, y, u)=u \cdot \mu(x), \sigma_{Y}(x, y, u)=\sigma^{\mathrm{T}}(x) u,
$$

Finally,

$$
G(x, y)=y-g(x) \text { for some Lipschitz continuous function } g: \mathbb{R}^{d} \longrightarrow \mathbb{R}_{+} \text {. }
$$

The process $X$ is thus defined by the stochastic differential equation

$$
d X_{t, x}(s)=\mu\left(X_{t, x}(s)\right) d s+\sigma\left(X_{t, x}(s)\right) d W_{s}, X_{t, x}(t)=x \in(0, \infty)^{d}
$$


and should be interpreted as the price process of $d$ risky securities. Here, we implicitly assume that the coefficients $\mu$ and $\sigma$ are such that $X_{t, x} \in(0, \infty)^{d} \mathbb{P}-$ a.s. for all initial conditions $(t, x) \in$ $[0, T] \times(0, \infty)^{d}$.

The control process $\nu$ is valued in $U=\mathbb{R}^{d}$, with components $\nu_{s}^{i}$ indicating the number of shares of the $i$-th security held in portfolio at time $s$. After the usual reduction of the interest rates to zero, it follows from the self-financing condition that the value of the portfolio is given by

$$
Y_{t, x, y}^{\nu}(s)=y+\int_{t}^{s} \nu_{r} \cdot d X_{t, x}(r), \quad s \geq t,
$$

which leads to the definitions in (4.3). The stochastic target problem $V(t, x)$ corresponds to the problem of super-hedging the contingent claim $g\left(X_{t, x}(T)\right)$, and $\bar{V}(t, x, p)$ is the corresponding quantile hedging problem. Note that the above assumptions ensure that $V$ is continuous and is given by $V(t, x)=\mathbb{E}^{\mathbb{Q}_{t, x}}\left[g\left(X_{t, x}(T)\right)\right]$ where $\mathbb{Q}_{t, x}$ is the $\mathbb{P}$-equivalent martingale measure defined by

$$
d \mathbb{Q}_{t, x} / d \mathbb{P}=\exp \left(-\frac{1}{2} \int_{t}^{T}\left|\lambda\left(X_{t, x}(s)\right)\right|^{2} d s-\int_{t}^{T} \lambda\left(X_{t, x}(s)\right) \cdot d W_{s}\right) .
$$

In particular, $V$ is a viscosity supersolution on $[0, T) \times(0, \infty)^{d}$ of

$$
0 \leq-\partial_{t} V-\frac{1}{2} \operatorname{Tr}\left[\sigma \sigma^{\mathrm{T}} D_{x x} V\right] \text {. }
$$

For later use, let us denote by $W^{\mathbb{Q}} t, x=W+\int_{t}^{\cdot} \lambda\left(X_{t, x}(s)\right) d s$ the $\mathbb{Q}_{t, x}$-Brownian motion defined on $[t, T]$.

In [5], the quantile hedging problem is solved in the general non-necessarilly Markov model of asset prices process, by means of the Neyman-Pearson lemma from mathematical statistics. In our Markov setting, we shall recover the solution of [5] by only using the supersolution property from the results of the previous sections.

First note that the conditions of Corollary 3.1, Theorem 3.1 and (i) of Proposition 3.2 are trivially satisfied. This implies that $\bar{V}_{*}$ is a viscosity supersolution of $[0, T) \times \overline{\mathbf{X}}$

$$
\begin{aligned}
0 & \leq-\partial_{t} \bar{V}_{*}+\bar{F}^{*} \bar{V}_{*} \\
& =-\partial_{t} \bar{V}_{*}-\frac{1}{2} \operatorname{Tr}\left[\sigma \sigma^{\mathrm{T}} D_{x x} \bar{V}_{*}\right]-\inf _{\alpha \in \mathbb{R}^{d}}\left(-\alpha\left(D_{p} \bar{V}_{*}\right)^{T} \sigma^{-1} \mu+\operatorname{Tr}\left[\sigma \alpha D_{x p} \bar{V}_{*}\right]+\frac{1}{2}|\alpha|^{2} D_{p p} \bar{V}_{*}\right)
\end{aligned}
$$

with the boundary conditions

$$
\bar{V}_{*}(\cdot, 1)=V \text { and } \bar{V}_{*}(\cdot, 0)=0 \text { on }[0, T] \times \mathbf{X} \text {, and } \bar{V}_{*}(T, x, p) \geq p g(x) \text { on } \mathbf{X} \times[0,1] .
$$

For sake of clarity, we extend $\bar{V}_{*}$ to $[0, T] \times \mathbf{X} \times \mathbb{R}$ by setting

$$
\bar{V}_{*}(\cdot, p):=0 \text { for } p<0 \text { and } \bar{V}_{*}(\cdot, p):=\infty \text { for } p>1 \text {. }
$$

The key idea for solving (4.6)-(4.7) is to introduce its Legendre-Fenchel dual with respect to the $p$-variable in order to remove the non-linearity in (4.6):

$$
v(t, x, q):=\sup _{p \in \mathbb{R}}\left\{p q-\bar{V}_{*}(t, x, p)\right\}, \quad(t, x, q) \in[0, T] \times(0, \infty)^{d} \times \mathbb{R} .
$$


Note that (4.8) and the second equality in (4.7) imply that

$$
v(\cdot, q)=\infty \text { for } q<0 \text { and } v(\cdot, q)=\sup _{p \in[0,1]}\left\{p q-\bar{V}_{*}(\cdot, p)\right\} \text { for } q>0
$$

Using the PDE characterization of $\bar{V}$ and $V$ above, we shall prove below that $v$ is an uppersemicontinuous viscosity subsolution on $[0, T) \times(0, \infty)^{d} \times(0, \infty)$ of

$$
-\partial_{t} v-\frac{1}{2} \operatorname{Tr}\left[\sigma \sigma^{\mathrm{T}} D_{x x} v\right]-\frac{1}{2}|\lambda|^{2} q^{2} D_{q q} v-\operatorname{Tr}\left[\sigma \lambda D_{x q} v\right] \leq 0
$$

with the boundary condition

$$
v(T, x, q) \leq(q-g(x))^{+}
$$

Since the above equation is linear, an explicit upper bound for $v$ is available from the Feynman-Kac representation result. Namely,

$$
v(t, x, q) \leq \bar{v}(t, x, q):=\mathbb{E}^{\mathbb{Q}_{t, x}}\left[\left(Q_{t, x, q}(T)-g\left(X_{t, x}(T)\right)\right)^{+}\right],
$$

on $[0, T] \times(0, \infty)^{d} \times(0, \infty)$, where the process $Q_{t, x, q}$ is defined by the dynamics

$$
\frac{d Q(s)}{Q(s)}=\lambda\left(X_{t, x}(s)\right) \cdot d W_{s}^{\mathbb{Q}_{t, x}}, Q_{t, x, q}(t)=q \in(0, \infty)
$$

Given the explicit representation of $\bar{v}$, we can now provide a lower bound to the primal function $\bar{V}$ by using (4.10). Clearly the function $\bar{v}$ is convex in $q$ and there is a unique solution $\hat{q}(t, x, p)$ to the equation

$$
\frac{\partial \bar{v}}{\partial q}(t, x, \hat{q})=\mathbb{E}^{\mathbb{Q} t, x}\left[Q_{t, x, 1}(T) \mathbf{1}_{\left\{Q_{t, x, \hat{q}}(T) \geq g\left(X_{t, x}(T)\right)\right\}}\right]=\mathbb{P}\left[Q_{t, x, \hat{q}}(T) \geq g\left(X_{t, x}(T)\right)\right]=p,
$$

where we have used the fact that $d \mathbb{P} / d \mathbb{Q}_{t, x}=Q_{t, x, 1}(T)$. It follows that the value function of the quantile hedging problem $\bar{V}$ admits the lower bound

$$
\begin{aligned}
\bar{V}(t, x, p) \geq \bar{V}_{*}(t, x, p) & \geq p \hat{q}(t, x, p)-\bar{v}(t, x, \hat{q}(t, x, p)) \\
& =\hat{q}(t, x, p)\left[p-\mathbb{E}^{\mathbb{Q}_{t, x}}\left[Q_{t, x, 1}(T) \mathbf{1}_{\left\{\hat{q}(t, x, p) Q_{t, x, 1}(T) \geq g\left(X_{t, x}(T)\right)\right\}}\right]\right] \\
& +\mathbb{E}^{\mathbb{Q} t, x}\left[g\left(X_{t, x}(T)\right) \mathbf{1}_{\left\{\hat{q}(t, x, p) Q_{t, x, 1}(T) \geq g\left(X_{t, x}(T)\right)\right\}}\right] \\
& =\mathbb{E}^{\mathbb{Q} t, x}\left[g\left(X_{t, x}(T)\right) \mathbf{1}_{\left\{\hat{q}(t, x, p) Q_{t, x, 1}(T) \geq g\left(X_{t, x}(T)\right)\right\}}\right]=: y(t, x, p) .
\end{aligned}
$$

On the other hand, it follows from the martingale representation theorem that we can find $\nu \in \mathcal{U}$ such that

$$
Y_{t, x, y(t, x, p)}^{\nu}(T) \geq g\left(X_{t, x}(T)\right) \mathbf{1}_{\left\{\hat{q}(t, x, p) Q_{t, x, 1}(T) \geq g\left(X_{t, x}(T)\right)\right\}} .
$$

Since, by (4.15), $\mathbb{P}\left[\hat{q}(t, x, p) Q_{t, x, 1}(T) \geq g\left(X_{t, x}(T)\right)\right]=p$, this implies that $\bar{V}(t, x, p)=y(t, x, p)$ which corresponds exactly to the solution of Föllmer and Leukert [5].

To conclude our argument, it remains to prove that $v$ is a viscosity subsolution of (4.11)-(4.12). 
Proof of (4.11)-(4.12). First note that the fact that $v$ is upper-semicontinuous on $[0, T] \times(0, \infty)^{d} \times$ $(0, \infty)$ follows from the lower-semicontinuity of $\bar{V}_{*}$ and the representation in the right-hand side of (4.10), which allows to reduce the computation of the sup to the compact set $[0,1]$. Moreover, the boundary condition (4.12) is an immediate consequence of the right-hand side inequality in (4.7) and (4.10) again.

We now turn to the PDE characterization inside the domain. Let $\varphi$ be a smooth function with bounded derivatives and $\left(t_{0}, x_{0}, q_{0}\right) \in[0, T) \times(0, \infty)^{d} \times(0, \infty)$ be a local maximizer of $v-\varphi$ such that $(v-\varphi)\left(t_{0}, x_{0}, q_{0}\right)=0$.

a. We first show that we can reduce to the case where the map $q \mapsto \varphi(\cdot, q)$ is strictly convex. Indeed, since $v$ is convex, we necessarily have $D_{q q} \varphi\left(t_{0}, x_{0}, q_{0}\right) \geq 0$. Given $\varepsilon, \eta>0$, we now define $\varphi_{\varepsilon, \eta}$ by $\varphi_{\varepsilon, \eta}(t, x, q):=\varphi(t, x, q)+\varepsilon\left|q-q_{0}\right|^{2}+\eta\left|q-q_{0}\right|^{2}\left(\left|q-q_{0}\right|^{2}+\left|t-t_{0}\right|^{2}+\left|x-x_{0}\right|^{2}\right)$. Note that $\left(t_{0}, x_{0}, q_{0}\right)$ is still a local maximizer of $U-\varphi_{\varepsilon, \eta}$. Since $D_{q q} \varphi\left(t_{0}, x_{0}, q_{0}\right) \geq 0$, we have $D_{q q} \varphi_{\varepsilon, \eta}\left(t_{0}, x_{0}, q_{0}\right) \geq 2 \varepsilon>0$. Since $\varphi$ has bounded derivatives, we can then choose $\eta$ large enough so that $D_{q q} \varphi_{\varepsilon, \eta}>0$. We next observe that, if $\varphi_{\varepsilon, \eta}$ satisfies (4.11) at $\left(t_{0}, x_{0}, q_{0}\right)$ for all $\varepsilon>0$, then (4.11) holds for $\varphi$ at this point too. This is due to the fact that the derivatives up to order two of $\varphi_{\varepsilon, \eta}$ at $\left(t_{0}, x_{0}, q_{0}\right)$ converge to the corresponding derivatives of $\varphi$ as $\varepsilon \rightarrow 0$.

b. From now on, we thus assume that the map $q \mapsto \varphi(\cdot, q)$ is strictly convex. Let $\tilde{\varphi}$ be the Fenchel transform of $\varphi$ with respect to $q$, i.e.

$$
\tilde{\varphi}(t, x, p):=\sup _{q \in \mathbb{R}}\{p q-\varphi(t, x, q)\} .
$$

Since $\varphi$ is strictly convex in $q$ and smooth on its domain, $\tilde{\varphi}$ is strictly convex in $p$ and smooth on its domain, see e.g. [8]. Moreover, we have

$$
\begin{aligned}
\varphi(t, x, q) & =\sup _{p \in \mathbb{R}}\{p q-\tilde{\varphi}(t, x, p)\} \\
& =J(t, x, q) q-\tilde{\varphi}(t, x, J(t, x, q)) \text { on }(0, T) \times(0, \infty)^{d} \times(0, \infty) \subset \operatorname{int}(\operatorname{dom}(\varphi))
\end{aligned}
$$

where $q \mapsto J(\cdot, q)$ denotes the inverse of $p \mapsto D_{p} \tilde{\varphi}(\cdot, p)$, recall that $\tilde{\varphi}$ is strictly convex in $p$.

We now deduce from the assumption $q_{0}>0$ and (4.10) that we can find $p_{0} \in[0,1]$ such that $v\left(t_{0}, x_{0}, q_{0}\right)=p_{0} q_{0}-\bar{V}_{*}\left(t_{0}, x_{0}, p_{0}\right)$ which, by using the very definition of $\left(t_{0}, x_{0}, p_{0}, q_{0}\right)$ and $v$, implies that

$$
\left(t_{0}, x_{0}, p_{0}\right) \text { is a local minimizer of } \bar{V}_{*}-\tilde{\varphi} \text { such that }\left(\bar{V}_{*}-\tilde{\varphi}\right)\left(t_{0}, x_{0}, p_{0}\right)=0
$$

and

$$
\varphi\left(t_{0}, x_{0}, q_{0}\right)=\sup _{p \in \mathbb{R}}\left\{p q_{0}-\tilde{\varphi}\left(t_{0}, x_{0}, p\right)\right\}=p_{0} q_{0}-\tilde{\varphi}\left(t_{0}, x_{0}, p_{0}\right) \text { with } p_{0}=J\left(t_{0}, x_{0}, q_{0}\right),
$$

where the last equality follows from (4.16) and the strict convexity of the map $p \mapsto p q_{0}-\tilde{\varphi}\left(t_{0}, x_{0}, p\right)$ in the domain of $\tilde{\varphi}$.

We conclude the proof by discussing three alternative cases depending on the value of $p_{0}$.

1. If $p_{0} \in(0,1)$, then (4.17) implies that $\tilde{\varphi}$ satisfies $(4.6)$ at $\left(t_{0}, x_{0}, p_{0}\right)$ and the required result 
follows by exploiting the link between the derivatives of $\tilde{\varphi}$ and the derivatives of its $p$-Fenchel transform $\varphi$, which can be deduced from (4.16).

2. If $p_{0}=1$, then the first boundary condition in (4.7) and (4.17) imply that $\left(t_{0}, x_{0}\right)$ is a local minimizer of $\bar{V}_{*}(\cdot, 1)-\tilde{\varphi}(\cdot, 1)=V-\tilde{\varphi}(\cdot, 1)$ such that $(V-\tilde{\varphi}(\cdot, 1))\left(t_{0}, x_{0}\right)=0$. This implies that $\tilde{\varphi}(\cdot, 1)$ satisfies $(4.5)$ at $\left(t_{0}, x_{0}\right)$ so that $\tilde{\varphi}$ satisfies $(4.6)$ for $\alpha=0$ at $\left(t_{0}, x_{0}, p_{0}\right)$. We can then conclude as in 1 . above.

3. If $p_{0}=0$, then the second boundary condition in (4.7) and (4.17) imply that $\left(t_{0}, x_{0}\right)$ is a local minimizer of $\bar{V}_{*}(\cdot, 0)-\tilde{\varphi}(\cdot, 0)=0-\tilde{\varphi}(\cdot, 0)$ such that $0-\tilde{\varphi}(\cdot, 0)\left(t_{0}, x_{0}\right)=0$. In particular, $\left(t_{0}, x_{0}\right)$ is a local maximum point for $\tilde{\varphi}(\cdot, 0)$ so that $\left(\partial_{t} \tilde{\varphi}, D_{x} \tilde{\varphi}\right)\left(t_{0}, x_{0}, 0\right)=0$ and $D_{x x} \tilde{\varphi}\left(t_{0}, x_{0}, 0\right)$ is negative semi-definite. This implies that $\tilde{\varphi}(\cdot, 0)$ satisfies $(4.5)$ at $\left(t_{0}, x_{0}\right)$ so that $\tilde{\varphi}$ satisfies $(4.6)$ at $\left(t_{0}, x_{0}, p_{0}\right)$, for $\alpha=0$. We can then argue as in the first case.

\section{Derivation of the DPE for singular stochastic target problems}

This section is dedicated to the proof of Theorems 2.1 and 2.2. We first recall the geometric dynamic programming principle of [10]. We next report the proof of the supersolution properties in subsections 5.1 and 5.2, and that of the subsolution properties in subsections 5.3 and 5.4.

Theorem 5.1 (Geometric Dynamic Programming Principle) Let $(t, x) \in[0, T) \times \mathbf{X}$ and $\theta$ be a $[s, T]$-valued stopping time. Then,

$$
V(t, x)=\inf \left\{y \in \mathbb{R}: Y_{t, x, y}^{\nu}(\theta) \geq V\left(\theta, X_{t, x}^{\nu}(\theta)\right) \text { a.s. for some } \nu \in \mathcal{U}\right\}
$$

The dynamic programming equation (2.7) is the infinitesimal analogue of the above geometric dynamic programming principle. The viscosity property stated in Theorem 2.1 is obtained in two steps. The super-solution property will be deduced from the following consequence of (5.1).

(GDP1) If $y>V(t, x)$, then there exists $\nu \in \mathcal{U}$ such that for all stopping time $\theta \leq T$

$$
Y_{t, x, y}^{\nu}(\theta) \geq V\left(\theta, X_{t, x}^{\nu}(\theta)\right)
$$

The sub-solution property will be proved using the following claim which is again implied by (5.1).

(GDP2) For every $y<V(t, x), \nu \in \mathcal{U}$, and all stopping time $\theta \leq T$,

$$
\mathbb{P}\left[Y_{t, x, y}^{\nu}(\theta)>V\left(\theta, X_{t, x}^{\nu}(\theta)\right)\right]<1 .
$$

Notice that (5.1) is equivalent to (GDP1)-(GDP2).

\subsection{The supersolution property on $[0, T) \times \mathbf{X}$}

This proof avoids delicate limit arguments of the supersolution derivation in [9] and [10] 
$\underline{\text { Step } 1}$ Let $\left(t_{0}, x_{0}\right) \in[0, T) \times \mathbf{X}$ and $\varphi$ be a smooth function such that

$$
\text { (strict) } \min _{[0, T) \times \mathbf{X}}\left(V_{*}-\varphi\right)=\left(V_{*}-\varphi\right)\left(t_{0}, x_{0}\right)=0 .
$$

Assume to the contrary that $\left(-\partial_{t} \varphi+F^{*} \varphi\right)\left(t_{0}, x_{0}\right)=:-2 \eta$ for some $\eta>0$, and let us work towards a contradiction. By definition of $F^{*}$, we may find $\varepsilon>0$, such that

$$
\mu_{Y}(x, y, u)-\mathcal{L}^{u} \varphi(t, x) \leq-\eta \text { for all } u \in \mathcal{N}_{\varepsilon}(x, y, D \varphi(t, x))
$$

$$
\text { and }(t, x, y) \in[0, T) \times \mathbf{X} \times \mathbb{R} \text { s.t. }(t, x) \in B_{\varepsilon}\left(t_{0}, x_{0}\right) \text { and }|y-\varphi(t, x)| \leq \varepsilon \text {, }
$$

where we recall that $B_{\varepsilon}\left(t_{0}, x_{0}\right)$ denotes the ball of radius $\varepsilon$ around $\left(t_{0}, x_{0}\right)$. Let $\partial_{p} B_{\varepsilon}\left(t_{0}, x_{0}\right):=$ $\left\{t_{0}+\varepsilon\right\} \times \operatorname{cl}\left(B_{\varepsilon}\left(t_{0}, x_{0}\right)\right) \cup\left[t_{0}, t_{0}+\varepsilon\right) \times \partial B_{\varepsilon}\left(x_{0}\right)$ denote the parabolic boundary of $B_{\varepsilon}\left(t_{0}, x_{0}\right)$ and observe that

$$
\zeta:=\min _{\partial_{p} B_{\varepsilon}\left(t_{0}, x_{0}\right)}\left(V_{*}-\varphi\right)>0
$$

since $\left(t_{0}, x_{0}\right)$ is a strict minimum.

Step 2 We now show that (5.4) and (5.5) lead to a contradiction to (GDP1). Let $\left(t_{n}, x_{n}\right)_{n}$ be a sequence in $[0, T) \times \mathbf{X}$ which converges to $\left(t_{0}, x_{0}\right)$ and such that $V\left(t_{n}, x_{n}\right) \rightarrow V_{*}\left(t_{0}, x_{0}\right)$. Set $y_{n}=V\left(t_{n}, x_{n}\right)+n^{-1}$ and observe that

$$
\gamma_{n}:=y_{n}-\varphi\left(t_{n}, x_{n}\right) \rightarrow 0 .
$$

For each $n \geq 1$, we have $y_{n}>V\left(t_{n}, x_{n}\right)$. Then, there exists some $\nu^{n} \in \mathcal{U}$ such that

$$
G\left(Z^{n}(T)\right) \geq 0 \quad \text { where } \quad Z^{n}:=\left(X^{n}, Y^{n}\right):=\left(X_{t_{n}, x_{n}}^{\nu^{n}}, Y_{t_{n}, x_{n}, y_{n}}^{\nu^{n}}\right) .
$$

We now define the stopping times

$$
\theta_{n}^{o}:=\left\{s \geq t_{n}:\left(s, X^{n}(s)\right) \notin B_{\varepsilon}\left(t_{0}, x_{0}\right)\right\}, \theta_{n}:=\left\{s \geq t_{n}:\left|Y^{n}(s)-\varphi\left(s, X^{n}(s)\right)\right| \geq \varepsilon\right\} \wedge \theta_{n}^{o}
$$

and set

$$
A_{n}:=\left\{s \in\left[t_{n}, \theta_{n}\right]: \mu_{Y}\left(Z^{n}(s), \nu_{s}^{n}\right)-\mathcal{L}^{\nu_{s}^{n}} \varphi\left(s, X^{n}(s)\right)>-\eta\right\} .
$$

Observe that (5.4) implies that the process

$$
\psi_{s}^{n}:=N^{\nu^{n}}\left(Z_{s}^{n}, D \varphi\left(s, X_{s}^{n}\right)\right) \quad \text { satisfies } \quad\left|\psi_{s}^{n}\right|>\varepsilon \text { for } s \in A_{n} .
$$

By (5.7) and (GDP1), it follows that

$$
Y^{n}\left(t \wedge \theta_{n}\right) \geq V\left(t \wedge \theta_{n}, X^{n}\left(t \wedge \theta_{n}\right)\right), \quad t \geq t_{n}
$$

Using the definition of $\zeta$ in (5.5) and $\theta_{n}$, this implies that

$$
\begin{aligned}
Y^{n}\left(t \wedge \theta_{n}\right) & \geq \varphi\left(t \wedge \theta_{n}, X^{n}\left(t \wedge \theta_{n}\right)\right)+\left(\zeta \mathbf{1}_{\left\{\theta_{n}^{o}=\theta_{n}\right\}}+\varepsilon \mathbf{1}_{\left\{\theta_{n}^{o}>\theta_{n}\right\}}\right) \mathbf{1}_{\left\{t=\theta_{n}\right\}} \\
& \geq \varphi\left(t \wedge \theta_{n}, X^{n}\left(t \wedge \theta_{n}\right)\right)+(\zeta \wedge \varepsilon) \mathbf{1}_{\left\{t=\theta_{n}\right\}}, \quad t \geq t_{n} .
\end{aligned}
$$


Since $\varphi$ is smooth, it follows from Itô's Lemma, (5.6), the definition of $\psi^{n},(2.8)$ and (5.8) that

$$
\begin{aligned}
-(\zeta \wedge \varepsilon) 1_{\left\{t<\theta_{n}\right\}} & \leq \gamma_{n}-(\zeta \wedge \varepsilon)+\int_{t_{n}}^{t \wedge \theta_{n}}\left\{\mu_{Y}\left(Z^{n}(s), \nu_{s}^{n}\right)-\mathcal{L}^{\nu_{s}^{n}} \varphi\left(s, X^{n}(s)\right)\right\} d s+\int_{t_{n}}^{t \wedge \theta_{n}} \psi_{s}^{n} \cdot d W_{s} \\
& \leq M_{t}^{n}:=\gamma_{n}-(\zeta \wedge \varepsilon)+\int_{t_{n}}^{t \wedge \theta_{n}} b_{s}^{n} d s+\int_{t_{n}}^{t \wedge \theta_{n}} \psi_{s}^{n} \cdot d W_{s}
\end{aligned}
$$

where we set $b_{s}^{n}:=\left\{\mu_{Y}\left(Z^{n}(s), \nu_{s}^{n}\right)-\mathcal{L}^{\nu_{s}^{n}} \varphi\left(s, X^{n}(s)\right)\right\} \mathbf{1}_{A_{n}}(s)$. Let $L^{n}$ be the exponential local martingale defined by $L_{t_{n}}^{n}=1$ and, for $s \geq t_{n}$,

$$
d L_{s}^{n}=-L_{s}^{n} b_{s}^{n}\left|\psi_{s}^{n}\right|^{-2} \psi_{s}^{n} \mathbf{1}_{A_{n}}(s) \cdot d W_{s},
$$

which is well defined by (5.9), (2.2) and our definition of the set of admissible controls $\mathcal{U}$.

By Itô's formula and (5.10), we see that $L^{n} M^{n}$ is a local martingale which is bounded from below by the submartingale $-(\zeta \wedge \varepsilon) L^{n}$. Then, $L^{n} M^{n}$ is a supermartingale, and it follows from (5.10) that

$$
0 \leq \mathbb{E}\left[L_{\theta_{n}}^{n} M_{\theta_{n}}^{n}\right] \leq L_{t_{n}}^{n} M_{t_{n}}^{n}=M_{t_{n}}^{n}=\gamma_{n}-\zeta \wedge \varepsilon,
$$

which contradicts (5.6) for $n$ large enough.

\subsection{The supersolution property on $\{T\} \times \mathbf{X}$}

Let $x_{0} \in \mathbf{X}$ and $\varphi$ be a smooth function such that

$$
(\text { strict }) \min _{\mathbf{X}}\left(V_{*}(T, \cdot)-\varphi\right)=\left(V_{*}(T, \cdot)-\varphi\right)\left(x_{0}\right)=0 .
$$

The fact that $\delta^{*} \varphi\left(x_{0}\right) \geq 0$ is deduced from (2.15) and the upper-semicontinuity of $\delta^{*}$ by standard arguments, see e.g. the proof of Lemma 5.2 in [9]. We now prove the second assertion. Assume that

$$
F^{*} \varphi\left(x_{0}\right)<\infty \text { and } \varphi\left(x_{0}\right)=V_{*}\left(T, x_{0}\right)<g_{*}\left(x_{0}\right)
$$

and let us work towards a contradiction. Since $V(T,)=$.$g by the definition of the problem, there$ is a constant $\eta>0$ such that $\varphi-V(T, \cdot) \leq \varphi-g_{*} \leq-\eta$ on $B_{\varepsilon}\left(x_{0}\right)$ for some $\varepsilon>0$. Since $x_{0}$ is a strict minimizer, $2 \zeta:=\min _{x \in \partial B_{\varepsilon}\left(x_{0}\right)} V_{*}(T, x)-\varphi(x)>0$ and it follows that there exists $r>0$ such that $V(t, x)-\varphi(x) \geq \zeta>0$ for all $(t, x) \in[T-r, T] \times \partial B_{\varepsilon}\left(x_{0}\right)$. Indeed, otherwise we could find $\left(t_{r}, x_{r}\right) \in[T-r, T] \times \partial B_{\varepsilon}\left(x_{0}\right)$ such that $V\left(t_{r}, x_{r}\right)-\varphi\left(x_{r}\right) \leq \zeta$, for each $r>0$. Sending $r \rightarrow 0$ would then lead to a contradiction since $\partial B_{\varepsilon}\left(x_{0}\right)$ is compact. Hence,

$$
V(t, x)-\varphi(x) \geq \zeta \wedge \eta>0 \text { for }(t, x) \in\left([T-r, T] \times \partial B_{\varepsilon}\left(x_{0}\right)\right) \cup\left(\{T\} \times B_{\varepsilon}\left(x_{0}\right)\right) .
$$

We now use the fact that $F^{*} \varphi\left(x_{0}\right)<\infty$ to deduce that, after possibly changing $\varepsilon>0$,

$$
\begin{gathered}
\mu_{Y}(x, y, u)-\mathcal{L}^{u} \varphi(x) \leq C \text { for all } u \in \mathcal{N}_{\varepsilon}(x, y, D \varphi(t, x)) \\
\text { and }(x, y) \in \mathbf{X} \times \mathbb{R} \text { s.t. } x \in B_{\varepsilon}\left(x_{0}\right) \text { and }|y-\varphi(x)| \leq \varepsilon,
\end{gathered}
$$


for some constant $C>0$. Let $\tilde{\varphi}(t, x):=\varphi(x)+C(t-T)$. Then, for sufficiently small $r>0$, we have

$$
\begin{gathered}
V(t, x)-\tilde{\varphi}(x) \geq \frac{1}{2}(\zeta \wedge \eta)>0 \text { for }(t, x) \in\left([T-r, T] \times \partial B_{\varepsilon}\left(x_{0}\right)\right) \cup\left(\{T\} \times B_{\varepsilon}\left(x_{0}\right)\right) \\
\text { and } \mu_{Y}(x, y, u)-\mathcal{L}^{u} \tilde{\varphi}(x) \leq C \text { for all } u \in \mathcal{N}_{\varepsilon}(x, y, D \tilde{\varphi}(t, x)) \\
(x, y) \in \mathbf{X} \times \mathbb{R} \text { s.t. } x \in B_{\varepsilon}\left(x_{0}\right) \text { and }|y-\tilde{\varphi}(x)| \leq \varepsilon .
\end{gathered}
$$

By following the arguments in Step 2 of Section 5.1, the latter inequalities lead to a contradiction of (GDP1).

\subsection{The sub-solution property on $[0, T) \times \mathbf{X}$}

We essentially adapt the arguments of [9] and [10]. Since our controls are not bounded and Assumption 2.1 is weaker that their assumptions, we provide the complete proof.

$\underline{\text { Step } 1}$ Let $\left(t_{0}, x_{0}\right) \in[0, T) \times \mathbf{X}$ and $\varphi$ be a smooth function such that

$$
0=\left(V^{*}-\varphi\right)\left(t_{0}, x_{0}\right)>\left(V^{*}-\varphi\right)(t, x), \quad\left(t_{0}, x_{0}\right) \neq(t, x) \in[0, T) \times \mathbf{X} .
$$

We have to show that $\left(-\partial_{t} \varphi+F_{*} \varphi\right)\left(t_{0}, x_{0}\right) \leq 0$. Assume to the contrary that

$$
4 \eta:=\left(-\partial_{t} \varphi+F_{*} \varphi\right)\left(t_{0}, x_{0}\right)>0 .
$$

By (2.9), we may find $\varepsilon>0$ such that

$$
\mu_{Y}(x, y, u)-\mathcal{L}^{u} \varphi(t, x) \geq 2 \eta \text { for some } u \in \mathcal{N}_{0}(x, y, D \varphi(t, x))
$$

for all $(t, x, y) \in[0, T) \times \mathbf{X} \times \mathbb{R}$ s.t. $(t, x) \in B_{\varepsilon}\left(t_{0}, x_{0}\right)$ and $|y-\varphi(t, x)| \leq \varepsilon$.

For $\varepsilon$ small enough, Assumption 2.1 then implies that

$$
\begin{aligned}
\alpha(t, x, y) & :=\mu_{Y}(x, y, \hat{\nu}(x, y, D \varphi(t, x)))-\mathcal{L}^{\hat{\nu}(x, y, D \varphi(t, x))} \varphi(t, x) \geq \eta \\
\text { for all }(t, x, y) & \in[0, T) \times \mathbf{X} \times \mathbb{R} \text { s.t. }(t, x) \in B_{\varepsilon}\left(t_{0}, x_{0}\right) \text { and }|y-\varphi(t, x)| \leq \varepsilon
\end{aligned}
$$

where $\hat{\nu}$ is a locally Lipschitz map satisfying

$$
\hat{\nu}(x, y, D \varphi(t, x)) \in \mathcal{N}_{0}(x, y, D \varphi(t, x)) \text { on } B_{\varepsilon}\left(t_{0}, x_{0}\right) .
$$

Observe that, since $\left(t_{0}, x_{0}\right)$ is a strict maximizer in $(5.12)$, we have

$$
-\zeta:=\max _{\partial_{p} B_{\varepsilon}\left(t_{0}, x_{0}\right)}\left(V^{*}-\varphi\right)<0,
$$

where $\partial_{p} B_{\varepsilon}\left(t_{0}, x_{0}\right):=\left\{t_{0}+\varepsilon\right\} \times \operatorname{cl}\left(B_{\varepsilon}\left(t_{0}, x_{0}\right)\right) \cup\left[t_{0}, t_{0}+\varepsilon\right) \times \partial B_{\varepsilon}\left(x_{0}\right)$ denotes the parabolic boundary of $B_{\varepsilon}\left(t_{0}, x_{0}\right)$.

Step 2 We now show that (5.13), (5.14) and (5.15) lead to a contradiction of (GDP2). Let $\left(t_{n}, x_{n}\right)_{n}$ be a sequence in $[0, T) \times \mathbf{X}$ which converges to $\left(t_{0}, x_{0}\right)$ and such that $V\left(t_{n}, x_{n}\right) \rightarrow$ $V^{*}\left(t_{0}, x_{0}\right)$. Set $y_{n}=V\left(t_{n}, x_{n}\right)-n^{-1}$ and observe that

$$
\gamma_{n}:=y_{n}-\varphi\left(t_{n}, x_{n}\right) \longrightarrow 0 \text {. }
$$


Let $Z^{n}:=\left(X^{n}, Y^{n}\right)$ denote the solution of the SDE (2.1) associated to the Markovian control $\hat{\nu}^{n}=\hat{\nu}\left(X^{n}, Y^{n}, D \varphi\left(\cdot, X^{n}\right)\right)$ and the initial condition $Z^{n}\left(t_{n}\right)=\left(x_{n}, y_{n}\right)$. Since $\hat{\nu}$ is locally Lipschitz, this solution is well-defined. We next define the stopping times

$$
\begin{aligned}
& \theta_{n}^{o}:=\inf \left\{s \geq t_{n}:\left(s, X^{n}(s)\right) \notin B_{\varepsilon}\left(t_{0}, x_{0}\right)\right\}, \\
& \theta_{n}:=\inf \left\{s \geq t_{n}:\left|Y^{n}(s)-\varphi\left(s, X^{n}(s)\right)\right| \geq \varepsilon\right\} \wedge \theta_{n}^{o} .
\end{aligned}
$$

Note that the first line in (5.13), (5.16) and a standard comparison theorem implies that $Y^{n}\left(\theta_{n}\right)-$ $\varphi\left(\theta_{n}, X^{n}\left(\theta_{n}\right)\right) \geq \varepsilon$ on $\left\{\left|Y^{n}\left(\theta_{n}\right)-\varphi\left(\theta_{n}, X^{n}\left(\theta_{n}\right)\right)\right| \geq \varepsilon\right\}$ for $n$ large enough. Since $V \leq V^{*} \leq \varphi$, we then deduce from (5.15) and the definition of $\theta_{n}$ that

$$
\begin{aligned}
& Y^{n}\left(\theta_{n}\right)-V\left(\theta_{n}, X^{n}\left(\theta^{n}\right)\right) \geq \mathbf{1}_{\left\{\theta_{n}<\theta_{n}^{o}\right\}}\left\{Y^{n}\left(\theta_{n}\right)-\varphi\left(\theta_{n}, X^{n}\left(\theta_{n}\right)\right)\right\} \\
& \quad+\mathbf{1}_{\left\{\theta_{n}=\theta_{n}^{o}\right\}}\left\{Y^{n}\left(\theta_{n}^{o}\right)-V^{*}\left(\theta_{n}^{o}, X^{n}\left(\theta_{n}^{o}\right)\right)\right\} \\
&= \varepsilon \mathbf{1}_{\left\{\theta_{n}<\theta_{n}^{o}\right\}}+\mathbf{1}_{\left\{\theta_{n}=\theta_{n}^{o}\right\}}\left\{Y^{n}\left(\theta_{n}^{o}\right)-V^{*}\left(\theta_{n}^{o}, X^{n}\left(\theta_{n}^{o}\right)\right)\right\} \\
& \geq \varepsilon \varepsilon \mathbf{1}_{\left\{\theta_{n}<\theta_{n}^{o}\right\}}+\mathbf{1}_{\left\{\theta_{n}=\theta_{n}^{o}\right\}}\left\{Y^{n}\left(\theta_{n}^{o}\right)+\zeta-\varphi\left(\theta_{n}^{o}, X^{n}\left(\theta_{n}^{o}\right)\right)\right\} \\
& \geq \varepsilon \wedge \zeta+\mathbf{1}_{\left\{\theta_{n}=\theta_{n}^{o}\right\}}\left\{Y^{n}\left(\theta_{n}^{o}\right)-\varphi\left(\theta^{o}, X^{n}\left(\theta_{n}^{o}\right)\right)\right\} .
\end{aligned}
$$

We continue by using Itô's formula:

$$
Y^{n}\left(\theta_{n}\right)-V\left(\theta_{n}, X^{n}\left(\theta^{n}\right)\right) \geq \varepsilon \wedge \zeta+\mathbf{1}_{\left\{\theta_{n}=\theta_{n}^{o}\right\}}\left(\gamma_{n}+\int_{t_{n}}^{\theta_{n}} \alpha\left(s, X_{s}^{n}, Y_{s}^{n}\right) d s\right)
$$

where the drift term $\alpha(\cdot) \geq \eta$ is defined in (5.13) and the diffusion coefficient vanishes by (5.14). Since $\varepsilon, \zeta>0$ and $\gamma_{n} \rightarrow 0$, this implies that

$$
Y^{n}\left(\theta_{n}\right) \geq V\left(\theta_{n}, X^{n}\left(\theta^{n}\right)\right) \text { for sufficiently large } n .
$$

Recalling that the initial position of the process $Y^{n}$ is $y_{n}=V\left(t_{n}, x_{n}\right)-n^{-1}<V\left(t_{n}, x_{n}\right)$, this is clearly in contradiction with part (GDP2) of the geometric dynamic programming principle.

\subsection{The subsolution property on $\{T\} \times \mathbf{X}$}

The proof combines arguments used in Section 5.3 and in Section 5.2 above. We only explain the main steps.

Let $x_{0} \in \mathbf{X}$ and $\varphi$ be a smooth function such that

$$
\text { (strict) } \max _{\mathbf{X}}\left(V^{*}(T, \cdot)-\varphi\right)=\left(V^{*}(T, \cdot)-\varphi\right)\left(x_{0}\right)=0
$$

Assume that

$$
\delta_{*} \varphi\left(x_{0}\right)>0 \text { and } \quad 0<4 \eta:=\varphi\left(x_{0}\right)-g^{*}\left(x_{0}\right)=V^{*}\left(T, x_{0}\right)-g^{*}\left(x_{0}\right) .
$$

By (2.14) and Assumption 2.1, we can find $r, \varepsilon>0$ and a locally Lipschitz map $\hat{\nu}$ satisfying

$$
\hat{\nu}(x, y, D \varphi(x)) \in \mathcal{N}_{0}(x, y, D \varphi(x))
$$

for all $(x, y) \in \mathbf{X} \times \mathbb{R}$ s.t. $x \in B_{r}\left(x_{0}\right)$ and $|y-\varphi(x)| \leq \varepsilon$. 
Set $\tilde{\varphi}(t, x):=\varphi(x)+\sqrt{T-t}$. Since $\partial_{t} \tilde{\varphi}(t, x) \rightarrow-\infty$ as $t \rightarrow T$, we deduce that, for $r>0$ small enough,

$$
\begin{gathered}
\alpha(x, y):=\mu_{Y}(x, y, \hat{\nu}(x, y, D \tilde{\varphi}(t, x)))-\mathcal{L}^{\hat{\nu}(x, y, D \tilde{\varphi}(t, x))} \tilde{\varphi}(t, x) \geq \eta \\
\text { for all }(t, x, y) \in[T-r, T) \mathbf{X} \times \mathbb{R} \text { s.t. } x \in B_{r}\left(x_{0}\right) \text { and }|y-\tilde{\varphi}(t, x)| \leq \varepsilon .
\end{gathered}
$$

Also observe that, since $V^{*}-\tilde{\varphi}$ is upper-semicontinuous and $\left(V^{*}(T, \cdot)-\varphi\right)\left(x_{0}\right)=0$, we can choose $r>0$ so that

$$
V(t, x) \leq \tilde{\varphi}(t, x)+\varepsilon / 2 \text { for all }(t, x) \in[T-r, T] \times B_{r}\left(x_{0}\right)
$$

Moreover, combining the identity $V\left(T, x_{0}\right)=g\left(x_{0}\right),(5.17),(5.19)$, the fact that $x_{0}$ achieves a strict maximum, and using similar arguments as those of Section 5.2 above, we see that

$$
V(t, x)-\tilde{\varphi}(t, x) \leq-\zeta \text { for all }(t, x) \in\left([T-r, T] \times \partial B_{r}\left(x_{0}\right)\right) \cup\left(\{T\} \times B_{r}\left(x_{0}\right)\right)
$$

for some $r, \varepsilon, \zeta>0$ small enough but so that the above inequalities still hold.

By following the arguments in Step 2 of Section 5.3, we see that (5.18), (5.19), (5.20) and (5.21) lead to a contradiction of (GDP2).

\section{Derivation of the boundary conditions for the stochastic target with controlled probability}

We now prove Theorem 3.1. The Dynkin operator associated to $\left(X^{\nu}, P^{\alpha}\right)$ will be denoted by

$$
\overline{\mathcal{L}}^{\bar{u}} \varphi(t, \bar{x}):=\partial_{t} \varphi(t, \bar{x})+\bar{\mu}(\bar{x}, \bar{u}) \cdot D \varphi(t, \bar{x})+\frac{1}{2} \operatorname{Tr}\left[\bar{\sigma} \bar{\sigma}^{\mathrm{T}}(\bar{x}, \bar{u}) D^{2} \varphi(t, \bar{x})\right] .
$$

\subsection{The endpoint $p=1$}

In order to prove that $\bar{V}_{*}(\cdot, 1)$ is a supersolution of $(2.10)-(2.19)$, it suffices to show that $\bar{V}_{*}(\cdot, 1)$ is a supersolution of

$$
\max \left\{\bar{V}_{*}(., 1)-V_{*},-\partial_{t} \bar{V}_{*}(., 1)+F^{*} \bar{V}_{*}(., 1)\right\} \geq 0 \quad \text { on } \quad[0, T) \times \mathbf{X},
$$

and that $\bar{V}_{*}(T, \cdot, 1)$ is a viscosity super-solution on $\mathbf{X}$ of

$$
\max \left\{\bar{V}_{*}(T, ., 1)-V_{*}(T, .), \min \left\{\left(\bar{V}_{*}(T, ., 1)-g_{*}\right) \mathbf{1}_{\left\{F^{*} \bar{V}_{*}(T, ., 1)<\infty\right\}}, \delta^{*} \bar{V}_{*}(T, \cdot, 1)\right\}\right\} \geq 0 .
$$

To convince ourself, let us show for instance that (6.1) implies (2.10). Let $\left(t_{0}, x_{0}\right)$ be a local minimizer of $\bar{V}_{*}(\cdot, 1)-\varphi$ for some smooth function $\varphi$. Then,

- either $\bar{V}_{*}\left(t_{0}, x_{0}, 1\right)<V_{*}\left(t_{0}, x_{0}\right)$ and $(2.10)$ holds for $\varphi$ at $\left(t_{0}, x_{0}\right)$,

- or $\bar{V}_{*}\left(t_{0}, x_{0}, 1\right)=V_{*}\left(t_{0}, x_{0}\right)$ so that $\left(t_{0}, x_{0}\right)$ is a local minimizer of $V_{*}-\varphi$, and (2.10) holds for $\varphi$ at $\left(t_{0}, x_{0}\right)$ by the viscosity property of $V_{*}$, see Theorem 2.1 . 
$\underline{\text { Step } 1}$ We first show that for any smooth function $\varphi$ on $[0, T] \times \mathbf{X} \times[0,1]$ and $\left(t_{0}, x_{0}\right) \in[0, T) \times \mathbf{X}$ such that

$$
\text { (strict) } \min _{[0, T) \times \mathbf{X} \times(0,1]}\left(\bar{V}_{*}-\varphi\right)=\left(\bar{V}_{*}-\varphi\right)\left(t_{0}, x_{0}, 1\right)=0,
$$

we have

$$
\max \left\{\varphi\left(t_{0}, x_{0}, 1\right)-V_{*}\left(t_{0}, x_{0}\right),\left(-\partial_{t} \varphi+\bar{F}^{*} \varphi\right)\left(t_{0}, x_{0}, 1\right)\right\} \geq 0
$$

If not, we can find $\eta, \varepsilon>0$ such that

$$
\begin{gathered}
\max \left\{\varphi(t, x, p)-V(t, x), \mu_{Y}(x, y, u)-\overline{\mathcal{L}}^{u, \alpha} \varphi(t, x, p)\right\} \leq-\eta \\
\text { for all }(u, \alpha) \in \overline{\mathcal{N}}_{\varepsilon}(x, y, D \varphi(t, x, p)) \text { and }(t, x, p, y) \in[0, T) \times \mathbf{X} \times(0,1] \times \mathbb{R}
\end{gathered}
$$

s.t. $(t, x, p) \in B_{\varepsilon}\left(t_{0}, x_{0}\right) \times[1-\varepsilon, 1]$ and $|y-\varphi(t, x, p)| \leq \varepsilon$.

Let $\left(t_{n}, x_{n}, p_{n}\right)_{n}$ be a sequence in $[0, T) \times \mathbf{X} \times(0,1)$ which converges to $\left(t_{0}, x_{0}, 1\right)$ and such that $\bar{V}\left(t_{n}, x_{n}, p_{n}\right) \rightarrow \bar{V}_{*}\left(t_{0}, x_{0}, 1\right)$. Set $y_{n}=\bar{V}\left(t_{n}, x_{n}, p_{n}\right)+n^{-1}$ and observe that

$$
\gamma_{n}:=y_{n}-\varphi\left(t_{n}, x_{n}\right) \rightarrow 0
$$

For each $n \geq 1$, we have $y_{n}>V\left(t_{n}, x_{n}\right)$. Then, there exists some $\bar{\nu}^{n}:=\left(\nu^{n}, \alpha^{n}\right) \in \overline{\mathcal{U}}$ such that

$$
\bar{G}\left(\bar{Z}^{n}(T)\right) \geq 0 \quad \text { where } \quad \bar{Z}^{n}:=\left(X^{n}, P^{n}, Y^{n}\right):=\left(X_{t_{n}, x_{n}}^{\nu^{n}}, P_{t_{n}, p_{n}}^{\alpha^{n}}, Y_{t_{n}, x_{n}, y_{n}}^{\nu^{n}}\right)
$$

We now define the stopping times

$$
\theta_{n}^{o}:=\left\{s \geq t_{n}:\left(s, X^{n}(s), P^{n}(s)\right) \in D\right\}, \theta_{n}:=\left\{s \geq t_{n}:\left|Y^{n}(s)-\varphi\left(s, X^{n}(s)\right)\right| \geq \varepsilon\right\} \wedge \theta_{n}^{o},
$$

where

$$
D:=\left(\partial_{p} B_{\varepsilon}\left(t_{0}, x_{0}\right) \times[1-\varepsilon, 1]\right) \cup\left(B_{\varepsilon}\left(t_{0}, x_{0}\right) \times\{1-\varepsilon, 1\}\right),
$$

and $\partial_{p} B_{\varepsilon}\left(t_{0}, x_{0}\right):=\left\{t_{0}+\varepsilon\right\} \times \operatorname{cl}\left(B_{\varepsilon}\left(t_{0}, x_{0}\right)\right) \cup\left[t_{0}, t_{0}+\varepsilon\right) \times \partial B_{\varepsilon}\left(x_{0}\right)$ denotes the parabolic boundary of $B_{\varepsilon}\left(t_{0}, x_{0}\right)$. It follows from (6.3) and (6.5) that

$$
\zeta:=\inf _{D}(\bar{V}-\varphi)>0 .
$$

By (6.6) and (GDP1), it follows that

$$
Y^{n}\left(\theta_{n}\right) \geq \bar{V}\left(\theta_{n}, X^{n}\left(\theta_{n}\right), P^{n}\left(\theta_{n}\right)\right) \geq \varphi\left(\theta_{n}, X^{n}\left(\theta_{n}\right), P^{n}\left(\theta_{n}\right)\right)
$$

where the second inequality follows from (6.3). Using the definition of $\theta_{n}$ and $\zeta>0$, this implies that

$$
Y^{n}\left(\theta_{n}\right) \geq \varphi\left(\theta_{n}, X^{n}\left(\theta_{n}\right), P^{n}\left(\theta_{n}\right)\right)+\zeta \wedge \varepsilon
$$

By arguing as in Section 5.1, this leads to a contradiction. 
$\underline{\text { Step } 2}$ We now show (6.1), i.e. for any smooth function $\varphi$ on $[0, T] \times \mathbf{X}$ and $\left(t_{0}, x_{0}\right) \in[0, T) \times \mathbf{X}$ such that

$$
\text { (strict) } \min _{[0, T) \times \mathbf{X}}\left(\bar{V}_{*}(\cdot, 1)-\varphi\right)=\left(\bar{V}_{*}(\cdot, 1)-\varphi\right)\left(t_{0}, x_{0}\right)=0
$$

we have

$$
\max \left\{\varphi\left(t_{0}, x_{0}\right)-V_{*}\left(t_{0}, x_{0}\right),\left(-\partial_{t} \varphi+F^{*} \varphi\right)\left(t_{0}, x_{0}\right)\right\} \geq 0 .
$$

a. For every $k>0$, we introduce the smooth function

$$
\varphi_{k}(t, x, p):=\varphi(t, x)-\left|x-x_{0}\right|^{4}-\left(t-t_{0}\right)^{2}-\psi_{k}(p)
$$

where, for some fixed $\rho>0$,

$$
\psi_{k}(p):=-\rho k \int_{p}^{1} \frac{e^{2 k}}{e^{k(r+1)}-e^{2 k+1}} d r, \quad k>0 .
$$

Observe that

$$
\begin{aligned}
& -2 \rho k \leq \psi_{k}^{\prime}(p)=\rho k \frac{e^{2 k}}{e^{k(p+1)}-e^{2 k+1}} \leq-\frac{\rho k}{2(e-1)} \quad \text { for } k \text { large enough } \\
& \psi_{k}^{\prime \prime}(p)=-\rho k^{2} \frac{e^{k(p+3)}}{\left(e^{k(p+1)}-e^{2 k+1}\right)^{2}}<0 \quad \text { for all } k>0 \\
& \lim _{k \rightarrow \infty} \frac{\left(\psi_{k}^{\prime}\left(p_{k}\right)\right)^{2}}{\left|\psi_{k}^{\prime \prime}\left(p_{k}\right)\right|}=\rho \quad \text { if }\left(p_{k}\right)_{k} \text { is a sequence in }[0,1] \text { s.t. } \lim _{k \rightarrow \infty} k\left(1-p_{k}\right)=0 .
\end{aligned}
$$

Let $\left(t_{k}, x_{k}, p_{k}\right)$ be a minimizer of $\bar{V}_{*}-\varphi_{k}$ on $[0, T] \times \bar{B}_{1}^{\mathbf{X}}\left(x_{0}\right) \times[0,1]$, where $\bar{B}_{1}^{\mathbf{X}}\left(x_{0}\right):=\bar{B}_{1}\left(x_{0}\right) \cap \mathbf{X}$ and $B_{1}\left(x_{0}\right)$ is the closed unit ball centered at $x_{0}$. Observe that, by definition of $\left(t_{k}, x_{k}, p_{k}\right)$ and $\left(t_{0}, x_{0}\right)$,

$$
\begin{aligned}
\left(\bar{V}_{*}-\varphi\right)\left(t_{0}, x_{0}, 1\right) & =\left(\bar{V}_{*}-\varphi_{k}\right)\left(t_{0}, x_{0}, 1\right) \\
& \geq\left(\bar{V}_{*}-\varphi_{k}\right)\left(t_{k}, x_{k}, p_{k}\right) \\
& =\left(\bar{V}_{*}-\varphi\right)\left(t_{k}, x_{k}, p_{k}\right)+\left|x_{k}-x_{0}\right|^{4}+\left(t_{k}-t_{0}\right)^{2}+\psi_{k}\left(p_{k}\right) \\
& \geq\left(\bar{V}_{*}-\varphi\right)\left(t_{k}, x_{k}, p_{k}\right)+\left|x_{k}-x_{0}\right|^{4}+\left(t_{k}-t_{0}\right)^{2}+\frac{\rho k}{2(e-1)}\left(1-p_{k}\right),
\end{aligned}
$$

where the last inequality follows from (6.9) for $k$ large enough and the fact that $\psi_{k}(1)=0$. Since $\bar{V}_{*} \geq 0$ by construction and $\varphi$ is bounded, this implies that the sequence $\left(t_{k}, x_{k}, p_{k}\right)_{k}$ is bounded and therefore converges to some $\left(t_{*}, x_{*}, p_{*}\right)$ up to a subsequence. Clearly, $p_{*}=1$, since otherwise we would have $k\left(1-p_{k}\right) \rightarrow \infty$. By definition of $\left(t_{0}, x_{0}\right)$, this implies that

$$
\begin{aligned}
\left(\bar{V}_{*}-\varphi\right)\left(t_{0}, x_{0}, 1\right) & \geq \liminf _{k \rightarrow \infty}\left(\bar{V}_{*}-\varphi_{k}\right)\left(t_{k}, x_{k}, p_{k}\right) \\
& \geq\left(\bar{V}_{*}-\varphi\right)\left(t_{*}, x_{*}, 1\right)+\left|x_{*}-x_{0}\right|^{4}+\left(t_{*}-t_{0}\right)^{2}+\liminf _{k \rightarrow \infty} \frac{\rho k}{2(e-1)}\left(1-p_{k}\right) \\
& \geq\left(\bar{V}_{*}-\varphi\right)\left(t_{0}, x_{0}, 1\right)+\left|x_{*}-x_{0}\right|^{4}+\left(t_{*}-t_{0}\right)^{2}+\liminf _{k \rightarrow \infty} \frac{\rho k}{2(e-1)}\left(1-p_{k}\right) .
\end{aligned}
$$


This shows that, after possibly passing to a subsequence,

$$
\left(t_{k}, x_{k}, p_{k}\right) \rightarrow\left(t_{0}, x_{0}, 1\right), k\left(1-p_{k}\right) \rightarrow 0 \quad \text { and } \quad \bar{V}_{*}\left(t_{k}, x_{k}, p_{k}\right) \rightarrow \bar{V}_{*}\left(t_{0}, x_{0}, 1\right)
$$

b. In order to prove (6.7), we assume that

$$
\bar{V}_{*}\left(t_{0}, x_{0}, 1\right)-V_{*}\left(t_{0}, x_{0}\right)<0,
$$

and we intend to prove that

$$
\left(-\partial_{t} \varphi+F^{*} \varphi\right)\left(t_{0}, x_{0}\right) \geq 0 .
$$

By the previous convergence results, it follows from (6.13) that the sequence $\left(t_{k}, x_{k}, p_{k}\right)$ of minimizers of the difference $\bar{V}_{*}-\varphi$ satisfy $\bar{V}_{*}\left(t_{k}, x_{k}, p_{k}\right)-V_{*}\left(t_{k}, x_{k}\right)<0$ after possibly passing to a subsequence. By Corollary 3.1 together with the result of Step 1, we then deduce that

$$
\left(-\partial_{t} \varphi_{k}+\bar{F}^{*} \varphi_{k}\right)\left(t_{k}, x_{k}, p_{k}\right) \geq 0 \text { for every } k>1 \text {. }
$$

Now observe that by (6.12)

$$
\begin{gathered}
\left(\partial_{t} \varphi_{k}, D_{x} \varphi_{k}, D_{x x}^{2} \varphi_{k}\right)\left(t_{k}, x_{k}, p_{k}\right) \rightarrow\left(\partial_{t} \varphi, D \varphi, D^{2} \varphi\right)\left(t_{0}, x_{0}\right) \quad \text { as } \quad k \rightarrow \infty \\
\left(D_{p} \varphi_{k}, D_{x p}^{2} \varphi_{k}, D_{p p}^{2} \varphi_{k}\right)\left(t_{k}, x_{k}, p_{k}\right)=\left(-\psi_{k}^{\prime}\left(p_{k}\right), 0,-\psi_{k}^{\prime \prime}\left(p_{k}\right)\right) \quad \text { for } \quad \text { every } k>1 .
\end{gathered}
$$

By definition of $\bar{F}^{*}$, we can then find sequences $\varepsilon_{k}>0, \bar{x}_{k}=\left(x_{k}^{0}, p_{k}^{0}\right) \in \mathbf{X} \times[0,1], y_{k} \geq 0$, $q_{k}=\left(q_{k}^{x}, q_{k}^{p}\right) \in \mathbb{R}^{d} \times \mathbb{R}$, and a symmetric matrix $A_{k} \in \mathbb{S}^{d+1}$ with rows $\left(A_{k}^{x x}, A_{k}^{x p}\right) \in \mathbb{S}^{d} \times \mathbb{R}^{d}$ and $\left(A_{k}^{x p \mathrm{~T}}, A_{k}^{p p}\right) \in \mathbb{R}^{d} \times \mathbb{R}$ such that

$$
\varepsilon_{k} \rightarrow 0, \bar{x}_{k} \rightarrow\left(x_{0}, 1\right), \quad \text { and } \quad\left|\left(y_{k}, q_{k}, A_{k}\right)-\left(\varphi, D \varphi, D^{2} \varphi_{k}\right)\left(t_{k}, x_{k}, p_{k}\right)\right| \leq k^{-1}
$$

and

$$
-\partial_{t} \varphi\left(t_{0}, x_{0}\right)+\bar{F}_{\varepsilon_{k}}\left(\bar{x}_{k}, y_{k}, q_{k}, A_{k}\right) \geq-k^{-1} .
$$

By the definition of $\bar{F}_{\varepsilon_{k}}$, we may find a maximizing sequence $\left(u_{k}, \alpha_{k}\right) \in \overline{\mathcal{N}}_{\varepsilon_{k}}\left(\bar{x}_{k}, y_{k}, q_{k}\right)$ such that

$$
\begin{aligned}
& -\partial_{t} \varphi\left(t_{0}, x_{0}\right)+\mu_{Y}\left(x_{k}^{0}, y_{k}, u_{k}\right)-\mu\left(x_{k}^{0}, u_{k}\right) \cdot q_{k}^{x} \\
& \quad-\frac{1}{2}\left(\operatorname{Tr}\left[\sigma \sigma^{\mathrm{T}}\left(x_{k}^{0}, u_{k}\right) A_{k}^{x x}\right]+\left|\alpha_{k}\right|^{2} A_{k}^{p p}+2 \sigma^{\mathrm{T}}\left(x_{k}^{0}, u_{k}\right) A_{k}^{x p} \cdot \alpha_{k}\right) \geq-2 k^{-1} .
\end{aligned}
$$

Observe that $\left(u_{k}, \alpha_{k}\right) \in \overline{\mathcal{N}}_{\varepsilon_{k}}\left(\bar{x}_{k}, y_{k}, q_{k}\right)$ implies that $u_{k} \in \mathcal{N}_{\varepsilon_{k}+\left|q_{k}^{p} \alpha_{k}\right|}\left(x_{k}^{0}, y_{k}, q_{k}^{x}\right)$. Then

$$
-\partial_{t} \varphi\left(t_{0}, x_{0}\right)+F_{\varepsilon_{k}+\left|q_{k}^{p} \alpha_{k}\right|}\left(x_{k}^{0}, y_{k}, q_{k}^{x}, A_{k}^{x x}\right) \geq-\frac{2}{k}+\frac{1}{2}\left|\alpha_{k}\right|^{2} A_{k}^{p p}+\sigma^{\mathrm{T}}\left(x_{k}^{0}, u_{k}\right) A_{k}^{x p} \cdot \alpha_{k},
$$

and we deduce from Assumption 3.3 that, for some constant $C>0$ (which may change from line to line),

$$
\begin{aligned}
C\left(1+\left|q_{k}^{p} \alpha_{k}\right|^{2}\right) & \geq \frac{1}{2} A_{k}^{p p}\left|\alpha_{k}\right|^{2}+\sigma^{\mathrm{T}}\left(x_{k}^{0}, u_{k}\right) A_{k}^{x p} \cdot \alpha_{k} \\
& \geq \frac{1}{2} A_{k}^{p p}\left|\alpha_{k}\right|^{2}+C\left|A_{k}^{x p}\right|\left|\alpha_{k}\right|
\end{aligned}
$$


where we used the condition that $\sup _{u \in U}|\sigma(x, u)|$ is locally bounded. From (6.9), (6.10), (6.11), (6.12), (6.15) and (6.16), it follows that

$$
A_{k}^{p p} \longrightarrow \infty, A_{k}^{x p} \longrightarrow 0, \text { and } \frac{\left(q_{k}^{p}\right)^{2}}{\left|A_{k}^{p p}\right|} \longrightarrow \rho \text { as } k \rightarrow \infty,
$$

and we deduce from (6.18) that, for small $\rho$,

$$
\left|\alpha_{k}\right|^{2}\left|A_{k}^{p p}\right| \leq C \text { and } \quad\left|\alpha_{k}\right|^{2}\left|q_{k}^{p}\right|^{2} \leq C \rho
$$

for some constant $C$. In view of (6.19), this implies that $\left|\alpha_{k}\right| \rightarrow 0$. We now return to (6.17) to deduce that

$$
-\partial_{t} \varphi\left(t_{0}, x_{0}\right)+F_{\varepsilon_{k}+\sqrt{C \rho}}\left(x_{k}^{0}, y_{k}, q_{k}^{x}, A_{k}^{x x}\right) \geq-\frac{2}{k}+\sigma^{\mathrm{T}}\left(x_{k}^{0}, u_{k}\right) A_{k}^{x p} \cdot \alpha_{k},
$$

since $A_{k}^{p p}>0$, and we obtain the required result (6.14) by sending $k \rightarrow \infty$ and then $\rho \rightarrow 0$, and recalling that $\left(\left|\alpha_{k}\right|, A_{k}^{x p}\right) \rightarrow 0$ and that $\sigma$ is locally bounded, uniformly in the control $u$.

Step 3 It remains to prove (6.2). The fact that $\bar{V}_{*}(T, \cdot, 1)$ is a viscosity supersolution of

$$
\max \left\{\bar{V}_{*}(T, ., 1)-V_{*}(T, .), \delta^{*} \bar{V}_{*}(T, ., 1)\right\} \geq 0
$$

is deduced from (6.7) of the previous step by using the same arguments as in Section 5.2. It remains to show that $\bar{V}_{*}(T, \cdot, 1)$ is a viscosity supersolution of

$$
\max \left\{\bar{V}_{*}(T, ., 1)-V_{*}(T, .),\left(\bar{V}_{*}(T, ., 1)-g_{*}\right) \mathbf{1}_{\left\{F^{*} \bar{V}_{*}(T, ., 1)<\infty\right\}}\right\} \geq 0 .
$$

By combining the arguments of Step 1 with those of Section 5.2, we first show that for any smooth function $\bar{\varphi}$ on $\mathbf{X} \times[0,1]$ and $x_{0} \in \mathbf{X}$ such that

$$
\text { (strict) } \min _{\mathbf{X} \times(0,1]}\left(\bar{V}_{*}(T, \cdot)-\bar{\varphi}\right)=\left(\bar{V}_{*}(T, \cdot)-\bar{\varphi}\right)\left(x_{0}, 1\right)=0,
$$

we have

$$
\max \left\{\bar{V}_{*}\left(T, x_{0}, 1\right)-V_{*}\left(T, x_{0}\right),\left(\bar{V}_{*}\left(T, x_{0}, 1\right)-\bar{g}_{*}\left(x_{0}\right)\right) \mathbf{1}_{\left\{\bar{F}^{*} \bar{\varphi}\left(x_{0}, 1\right)<\infty\right\}}\right\} \geq 0 .
$$

We then consider a smooth function $\varphi$ on $\mathbf{X}$ and $x_{0} \in \mathbf{X}$ such that

$$
\text { (strict) } \min _{\mathbf{X}}\left(\bar{V}_{*}(T, \cdot, 1)-\varphi\right)=\left(\bar{V}_{*}(T, \cdot, 1)-\varphi\right)\left(x_{0}\right)=0
$$

and

$$
\bar{\varphi}\left(T, x_{0}\right)<V_{*}\left(T, x_{0}\right), \quad F^{*} \varphi\left(T, x_{0}\right)<\infty .
$$

We follow the construction of Step 2 of modified test functions

$$
\varphi_{k}(x, p):=\varphi(x)-\left|x-x_{0}\right|^{4}-\psi_{k}(p),
$$

where $\psi_{k}$ is defined in (6.8). As in the above Step 2, we prove that the difference $\bar{V}_{*}(T,)-.\varphi_{k}$ has a local minimizer $\bar{x}_{k}=\left(x_{k}, p_{k}\right)$ satisfying all estimates derived in the above Step 2 (forgetting about the $t$ variable). In particular, since $F^{*} \varphi\left(\bar{x}_{k}\right) \leq C$ for some constant $C>0$ independent of $k$ by (6.21), we deduce from the same estimates than in Step 2 that $\bar{F}^{*} \varphi\left(\bar{x}_{k}\right) \leq 2 C$ for all large $k$. It then follows from Corollary 3.2, (6.20) and (6.21) that $\bar{V}_{*}\left(T, \bar{x}_{k}\right) \geq \bar{g}_{*}\left(\bar{x}_{k}\right)$. Sending $k \rightarrow \infty$, this provides $\bar{V}_{*}\left(T, x_{0}, 1\right) \geq \bar{g}_{*}\left(x_{0}, 1\right)$, and the proof is completed by observing that $\bar{g}_{*}\left(x_{0}, 1\right)=g_{*}\left(x_{0}\right)$. 


\subsection{The endpoint $p=0$}

We organize the proof in three steps. As in the previous subsection, Steps 1 and 2 focus on $t<T$ while Step 3 concentrates on $t=T$.

$\underline{\text { Step } 1}$ We first show that for any smooth function $\bar{\varphi}$ on $[0, T) \times \mathbf{X} \times[0,1]$ and $\left(t_{1}, x_{1}\right) \in[0, T) \times \mathbf{X}$ such that

$$
\text { (strict) } \max _{[0, T) \times \mathbf{X} \times[0,1]}\left(\bar{V}^{*}-\bar{\varphi}\right)=\left(\bar{V}^{*}-\bar{\varphi}\right)\left(t_{1}, x_{1}, 0\right)=0,
$$

we have

$$
\min \left\{\bar{V}^{*},-\partial_{t} \bar{\varphi}+\bar{F}_{*} \bar{\varphi}\right\}\left(t_{1}, x_{1}, 0\right) \leq 0 .
$$

The proof is very similar to that of Subsection 5.3 up to the modification explained in the proof of Corollary 3.1 and the fact that we have to handle the state constraint $p=0$. For completeness, we report here the entire argument. Assume on the contrary that

$$
4 \eta:=\min \left\{\bar{V}^{*},\left(-\partial_{t} \bar{\varphi}+\bar{F}_{*} \bar{\varphi}\right)\left(t_{0}, x_{0}, 0\right)\right\}>0
$$

i.e., for some $\varepsilon>0$,

$$
\begin{gathered}
\min \left\{\bar{\varphi}(t, \bar{x}), \mu_{Y}(\bar{x}, y, \bar{u})-\overline{\mathcal{L}}^{\bar{u}} \bar{\varphi}(t, \bar{x})\right\} \geq 2 \eta \text { for some } \bar{u} \in \overline{\mathcal{N}}_{0}(\bar{x}, y, D \bar{\varphi}(t, \bar{x})) \\
\text { for all }(t, \bar{x}, y) \in[0, T) \times \overline{\mathbf{X}} \times \mathbb{R} \text { s.t. }(t, \bar{x}) \in B_{\varepsilon}\left(t_{0}, x_{0}\right) \times[0, \varepsilon],|y-\bar{\varphi}(t, \bar{x})| \leq \varepsilon
\end{gathered}
$$

Assumption 3.1 implies that

$$
\begin{gathered}
\alpha(t, x, y):=\min \left\{\bar{\varphi}(t, \bar{x}), \mu_{Y}(\bar{x}, y, \bar{\nu}(\bar{x}, y, D \bar{\varphi}(t, \bar{x})))-\overline{\mathcal{L}}^{\bar{\nu}(\bar{x}, y, D \bar{\varphi}(t, \bar{x}))} \bar{\varphi}(t, \bar{x})\right\} \geq \eta \\
\quad \text { for }(t, \bar{x}, y) \in[0, T] \times \overline{\mathbf{X}} \times \mathbb{R} \text { s.t. }(t, \bar{x}) \in B_{\varepsilon}\left(t_{0}, x_{0}\right) \times[0, \varepsilon],|y-\bar{\varphi}(t, \bar{x})| \leq \varepsilon
\end{gathered}
$$

where $\bar{\nu}$ is a locally Lipschitz map satisfying

$$
\bar{\nu}(\bar{x}, y, D \bar{\varphi}(t, \bar{x})) \in \overline{\mathcal{N}}_{0}(\bar{x}, y, D \bar{\varphi}(t, \bar{x})) \text { on } B_{\varepsilon}\left(t_{0}, x_{0}\right) \times[0, \varepsilon] .
$$

Observe that since $\left(t_{1}, x_{1}\right)$ is a strict maximizer in $(6.22)$, we have

$$
-\zeta:=\max _{D}\left(\bar{V}^{*}-\bar{\varphi}\right)<0, \text { where } D:=\left(\partial_{p} B_{\varepsilon}\left(t_{0}, x_{0}\right) \times[0, \varepsilon]\right) \cup\left(B_{\varepsilon}\left(t_{0}, x_{0}\right) \times\{\varepsilon\}\right)
$$

Also, we deduce from $(6.24)$ and the fact that $\bar{V}(\cdot, 0)=0$ by definition:

$$
0>-\eta \geq \max _{B_{\varepsilon}\left(t_{0}, x_{0}\right)}(\bar{V}-\bar{\varphi})(\cdot, 0)
$$

By following the arguments in Step 2 of Section 5.3, we see that (6.25), (6.26), (6.27) and (6.28) lead to a contradiction of (GDP2).

$\underline{\text { Step } 2}$ Let $\varphi$ be a smooth test function on $[0, T] \times \mathbf{X}$ and $\left(t_{0}, x_{0}\right) \in[0, T) \times \mathbf{X}$ be such that

$$
\text { (strict) } \max _{[0, T) \times \mathbf{X}}\left(\bar{V}^{*}-\varphi\right)(\cdot, 0)=\left(\bar{V}^{*}-\varphi\right)\left(t_{0}, x_{0}, 0\right)=0
$$


By definition, we have $\bar{V}^{*}\left(t_{0}, x_{0}, 0\right) \geq 0$. Let us assume that

$$
\bar{V}^{*}\left(t_{0}, x_{0}, 0\right)>0
$$

and work towards a contradiction. Recall the function $\psi_{k}$ of (6.8), and define

$$
\varphi_{k}(t, x, p):=\varphi(t, x)+\left|x-x_{0}\right|^{4}+\left(t-t_{0}\right)^{2}+\psi_{k}(1-p) .
$$

Arguing as in Step 2 of the previous subsection, we see that the difference $\bar{V}^{*}-\varphi_{k}$ has a local maximizer $\left(t_{k}, x_{k}, p_{k}\right)$ on $\operatorname{cl}([0, T] \times \mathbf{X} \times[0,1])$ satisfying

$$
\left(t_{k}, x_{k}, p_{k}\right) \rightarrow\left(t_{0}, x_{0}, 0\right), k p_{k} \rightarrow 0 \quad \text { and } \quad \bar{V}^{*}\left(t_{k}, x_{k}, p_{k}\right) \rightarrow \bar{V}^{*}\left(t_{0}, x_{0}, 0\right)
$$

so that

$$
\begin{aligned}
& \left(\partial_{t} \varphi_{k}, D_{x} \varphi_{k}, D_{x x}^{2} \varphi_{k}\right)\left(t_{k}, x_{k}, p_{k}\right) \longrightarrow\left(\partial_{t} \varphi, D \varphi, D^{2} \varphi\right)\left(t_{0}, x_{0}\right) \\
& \left(D_{p} \varphi_{k}, D_{x p}^{2} \varphi_{k}, D_{p p}^{2} \varphi_{k}\right)\left(t_{k}, x_{k}, p_{k}\right)=\left(-\psi_{k}^{\prime}\left(1-p_{k}\right), 0, \psi_{k}^{\prime \prime}\left(1-p_{k}\right)\right) .
\end{aligned}
$$

Since $\bar{V}^{*}\left(t_{0}, x_{0}, 0\right)>0$, we have $\bar{V}^{*}\left(t_{k}, x_{k}, p_{k}\right)>0$ for all $k$, after possibly passing to a subsequence. Then, it follows from Corollary 3.1 and Step 1 that

$$
\left(-\partial_{t} \varphi_{k}+\bar{F}_{*} \varphi_{k}\right)\left(t_{k}, x_{k}, p_{k}\right) \leq 0 \text { for all } k>1
$$

By the definition of $\bar{F}_{*}$, we can then find sequences $\varepsilon_{k}>0, \bar{x}_{k}=\left(x_{k}^{0}, p_{k}^{0}\right) \in \mathbf{X} \times[0,1], y_{k} \geq 0$, $q_{k}=\left(q_{k}^{x}, q_{k}^{p}\right) \in \mathbb{R}^{d} \times \mathbb{R}$, and a symmetric matrix $A_{k} \in \mathbb{S}^{d+1}$ with rows $\left(A_{k}^{x x}, A_{k}^{x p}\right) \in \mathbb{S}^{d} \times \mathbb{R}^{d}$ and $\left(A_{k}^{x p \mathrm{~T}}, A_{k}^{p p}\right) \in \mathbb{R}^{d} \times \mathbb{R}$ such that

$$
\varepsilon_{k} \rightarrow 0, \bar{x}_{k} \rightarrow\left(x_{0}, 1\right), \quad \text { and } \quad\left|\left(y_{k}, q_{k}, A_{k}\right)-\left(\varphi, D \varphi, D^{2} \varphi_{k}\right)\left(t_{k}, x_{k}, p_{k}\right)\right| \leq k^{-1}
$$

and

$$
-\partial_{t} \varphi\left(t_{0}, x_{0}\right)+\bar{F}_{\varepsilon_{k}}\left(\bar{x}_{k}, y_{k}, q_{k}, A_{k}\right) \leq k^{-1}
$$

i.e. for every $(u, \alpha) \in \overline{\mathcal{N}}_{\varepsilon_{k}}\left(\bar{x}_{k}, y_{k}, q_{k}\right)$ :

$$
\begin{aligned}
& -\partial_{t} \varphi\left(t_{0}, x_{0}\right)+\mu_{Y}\left(x_{k}^{0}, y_{k}, u\right)-\mu\left(x_{k}^{0}, u\right) \cdot q_{k}^{x} \\
& \quad-\frac{1}{2}\left(\operatorname{Tr}\left[\sigma \sigma^{\mathrm{T}}\left(x_{k}^{0}, u\right) A_{k}^{x x}\right]+|\alpha|^{2} A_{k}^{p p}+2 \sigma^{\mathrm{T}}\left(x_{k}^{0}, u\right) A_{k}^{x p} \cdot \alpha\right) \leq k^{-1} .
\end{aligned}
$$

Observe that (6.9), (6.10), (6.11), (6.30), and (6.31) imply that

$$
A_{k}^{p p}<0,\left|q_{k}^{p}\right|>0, \text { for large } k, \lim _{k \rightarrow \infty} A_{k}^{x p}=0, \text { and } \lim _{k \rightarrow \infty} \frac{\left(q_{k}^{p}\right)^{2}}{\left|A_{k}^{p p}\right|}=\rho .
$$

Now let $u \in U$ be an arbitrary control, and define $\alpha^{k}:=N^{u}\left(x_{k}^{0}, y_{k}, q_{k}^{x}\right) / q_{k}^{p}$, so that $\left(u, \alpha_{k}\right) \in$ $\overline{\mathcal{N}}_{\varepsilon_{k}}\left(\bar{x}_{k}, y_{k}, q_{k}\right)$, and it follows from (6.33) that

$$
\frac{\left|A_{k}^{p p}\right|}{\left(q_{k}^{p}\right)^{2}}\left|N^{u}\left(x_{k}^{0}, y_{k}, q_{k}^{x}\right)\right|^{2} \leq C
$$


for some $C>0$ independent of $k$ and $\rho$. Sending $k \rightarrow \infty$ in the above inequality, we then deduce from (6.32) and (6.34) that

$$
\rho^{-1}\left|N^{u}\left(x_{0}, \varphi\left(t_{0}, x_{0}\right), D \varphi\left(t_{0}, x_{0}\right)\right)\right|^{2} \leq C .
$$

Since $\rho>0$ can be chosen arbitrarily close to 0 , this shows that $N^{u}\left(x_{0}, \varphi\left(t_{0}, x_{0}\right), D \varphi\left(t_{0}, x_{0}\right)\right)=0$, and the arbitrariness of $u \in U$ is in contradiction with Assumption 3.2. Hence, (6.29) does not hold and therefore $\bar{V}^{*}(\cdot, 0)=0$ on $[0, T) \times \mathbf{X}$.

$\underline{\text { Step } 3}$ We finally show that $\bar{V}_{*}(T, \cdot, 0)=0$ on $\mathbf{X}$. Since $\bar{V}^{*}(t, x, 0)=0$ for $t<T$ and $x \in \mathbf{X}$, we can find a sequence $\left(t_{n}, x_{n}, p_{n}\right)_{n}$ in $[0, T) \times \mathbf{X} \times(0,1)$ such that $\left(t_{n}, x_{n}, p_{n}\right) \rightarrow(T, x, 0)$ and $0 \leq \bar{V}\left(t_{n}, x_{n}, p_{n}\right) \leq 1 / n$ for all $n \geq 0$. Passing to the limit leads to the required result.

\section{References}

[1] Bentahar I. and B. Bouchard (2006). Barrier option hedging under constraints: a viscosity approach, SIAM Journal on Control and Optimization, 45 (5), 1846-1874.

[2] Bouchard B. (2002), Stochastic Targets with Mixed diffusion processes, Stochastic Processes and their Applications, 101, 273-302.

[3] Crandall M.G., H. Ishii and P.-L. Lions (1992), User's guide to viscosity solutions of second order Partial Differential Equations, Amer. Math. Soc., 27, 1-67.

[4] Cvitanic J., H. Pham and N. Touzi (1999), A closed-form solution to the problem of superreplication under transaction costs, Finance and Stochastics, 3, 35-54.

[5] Föllmer H. and P. Leukert (1999), Quantile Hedging. Finance and Stochastics, 3, 3, 251-273.

[6] Föllmer H. et P. Leukert (2000), Efficient hedging : cost versus shortfall risk, Finance and Stochastics , 4, 117-146.

[7] Lions P.-L. and J.-M. Lasry (2004), Large Investor Trading Impacts on Volatility, ParisPrinceton Lectures on Mathematical Finance, Lecture Notes in Mathematics, 1919, 173-190.

[8] Rockafellar R.T. (1970), Convex Analysis. Princeton University Press, Princeton, NJ.

[9] Soner H. M. and N. Touzi (2002), Stochastic target problems, dynamic programming and viscosity solutions, SIAM Journal on Control and Optimization, 41, 404-424.

[10] Soner H. M. and N. Touzi (2002), Dynamic programming for stochastic target problems and geometric flows, Journal of the European Mathematical Society, 4, 201-236.

[11] Touzi N. (2000), Direct characterization of the value of super-replication under stochastic volatility and portfolio constraints, Stochastic Processes and their Applications, 88, 305-328. 